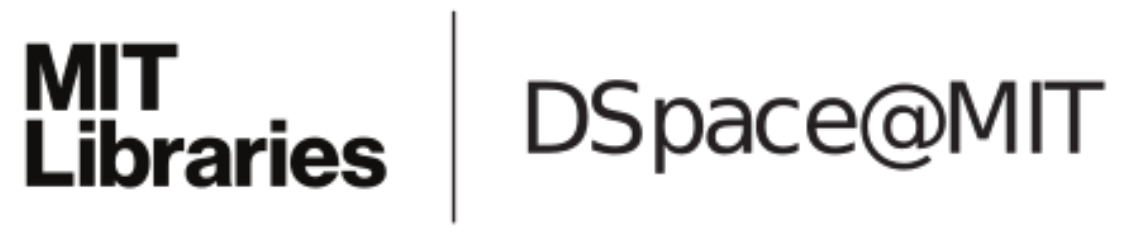

\author{
MIT Open Access Articles
}

Lignin-KMC: A Toolkit for Simulating Lignin Biosynthesis

The MIT Faculty has made this article openly available. Please share how this access benefits you. Your story matters.

As Published: $10.1021 /$ ACSSUSCHEMENG.9B03534

Publisher: American Chemical Society (ACS)

Persistent URL: https://hdl.handle.net/1721.1/136531

Version: Original manuscript: author's manuscript prior to formal peer review

Terms of use: Creative Commons Attribution-Noncommercial-Share Alike 


\title{
LIGNIN-KMC: A Toolkit for Simulating Lignin Biosynthesis
}

\author{
Michael J. Orella, ${ }^{1}$ Terry Z. H. Gani, ${ }^{1}$ Joshua V. Vermaas, ${ }^{2}$ Michael L. Stone, ${ }^{1}$ Eric M. Anderson, ${ }^{1}$ Gregg T. \\ Beckham, ${ }^{3}$ Fikile R. Brushett, ${ }^{* 1}$ Yuriy Román-Leshkov*,1 \\ ${ }^{1}$ Department of Chemical Engineering, Massachusetts Institute of Technology, Cambridge, MA 02139, USA \\ ${ }^{2}$ Biosciences Center, National Renewable Energy Laboratory, Golden, CO 80401, USA \\ ${ }^{3}$ National Bioenergy Center, National Renewable Energy Laboratory, Golden, CO 80401, USA
}

Abstract

Lignin is an abundant biopolymer of phenylpropanoid monomers that is critical for plant structure and function. Based on the abundance of lignin in the biosphere and interest in lignin valorization, a more comprehensive understanding of lignin biosynthesis is imperative. Here we present an open-source software toolkit, LIGNIN-KMC, that combines kinetic Monte Carlo and first-principles calculations of radical coupling events, which enables modeling of lignin biosynthesis in silico. Specifically, lignification is simulated using the Gillespie algorithm with a graph representation of the lignin fragments and with rates derived from density functional theory calculations of individual fragment couplings. Using this approach, we confirm experimental findings regarding the impact of lignification conditions on final polymer structure and identify new features of fundamental interest to plant cell wall biosynthesis including (1) the monolignol supply rate under in planta lignification conditions likely varies as a function of evolutionary stresses; (2) under conditions of low monolignol supply rates, increasing the fraction of sinapyl alcohol increases the depolymerization yield of monomers upon ether bond cleavage; and (3) by including calculated energetics of caffeyl alcohol homopolymers, the model accurately predicts C-lignin structures. These examples not only highlight the robustness of our modeling framework, but also motivate future studies of new lignin types, unexplored monolignol chemistries, and lignin structure predictions, all with an overarching aim of developing a more comprehensive, interested in this important biopolymer. 


\section{Introduction}

27 Lignin is an alkyl-aromatic polymer that gives structural integrity to plant matter and protects it from microbial attack. ${ }^{1-}$

$28{ }^{5}$ As a highly abundant natural biopolymer, lignin has substantial promise as a renewable feedstock for chemical 29 synthesis of aromatics. ${ }^{6-10}$ To economically convert lignocellulosic biopolymers, effective utilization of both 30 polysaccharides and lignins is imperative as wasted feedstocks rapidly decrease the profitability of any biorefinery 31 venture. ${ }^{11}$ In current generation biorefineries, we are well equipped to upgrade the polysaccharide fraction through a 32 number of catalytic ${ }^{12-17}$ and biological ${ }^{18-20}$ strategies. However, our understanding of lignin biosynthesis remains 33 incomplete, limiting our ability to fully valorize lignin. A better quantitative understanding of the complex native lignin 34 structure can enable the advancement, optimization, and, ultimately, deployment of recently proposed 35 depolymerization techniques, ${ }^{8,21-23}$ improve the suite of natural lignin-based polymer products, ${ }^{24-29}$ provide a starting 36 point for analysis of higher molecular weight lignin fragments, ${ }^{30-34}$ and guide the efforts of plant biologists interested 37 in genetically modifying plants to produce designed lignins. ${ }^{35-39}$

38 Lignin biosynthesis, or lignification, begins in the cytoplasm where a series of metabolic pathways convert 39 phenylalanine and tyrosine into primarily coniferyl alcohol (G-unit), sinapyl alcohol (S-unit), and $p$-coumaryl alcohol 40 (H-unit), which are collectively known as the monolignols., ${ }^{4,5,40-46}$ These molecules are subsequently transported to 41 the cell wall through a number of mechanisms, ${ }^{47,48}$ where peroxidase and laccase enzymes mediate their oxidation into 42 phenolic radicals. The radicals delocalize over the aromatic structure, resulting in coupling reactions that generate a 43 complex phenolic polymer network containing a variety of intermolecular C-O or C-C bonds. ${ }^{1,2}$ Figure 1 illustrates the 44 most common chemical linkages found in native lignin fragments and their associated nomenclature which will be 45 used throughout the remainder of this manuscript. 

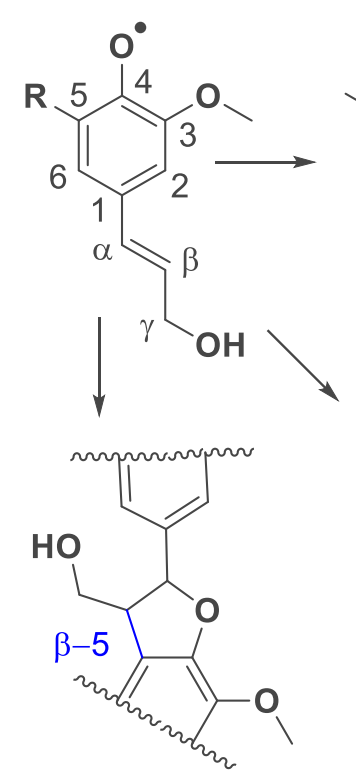
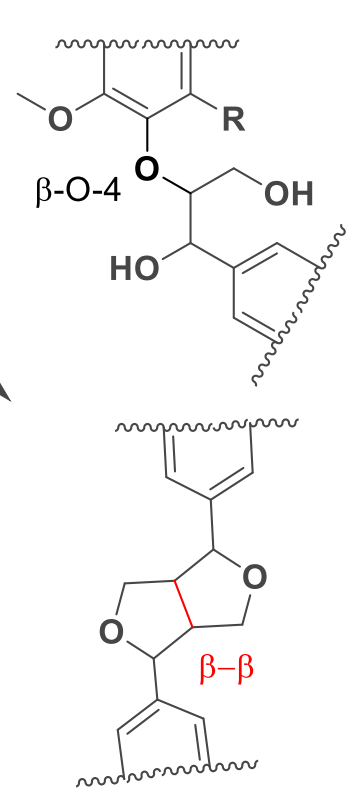
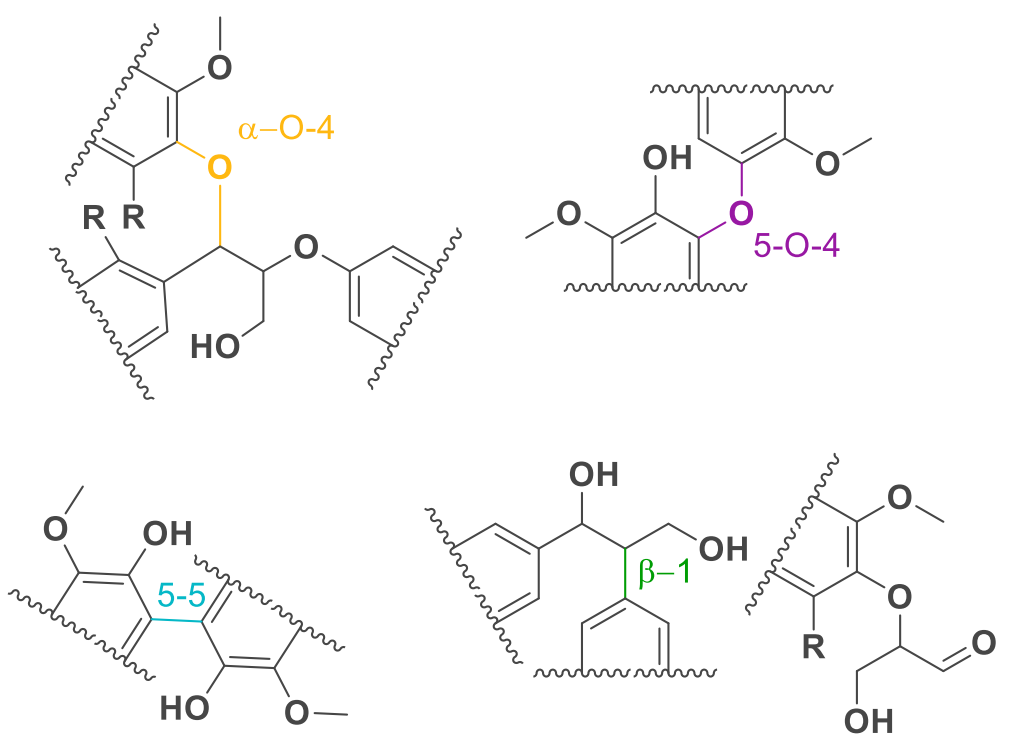

Figure 1: Lignification reactions that can occur in $H, S$, and $G$ lignin between oxidized fragments. These connections represent the linkages that are most commonly found in native lignins and the linkages represented in the LIGNIN-KMC code. The characteristic bond for each linkage is color coded, and this color code is used throughout the main text.

Over the years, multiple research groups have attempted to model the lignification process to obtain a structural understanding via a variety of stochastic methods parameterized by experimental data on bond distributions. For example, SIMREL, one of the earliest models of lignification, added monomers to growing chains of lignin so long as the chains conformed to experimentally measured lignins. ${ }^{49}$ Similarly, to better understand the geometry of lignin within the middle lamella, Jurasek's model of lignification in the cell wall relied on bond formation probabilities obtained from experimental data. ${ }^{50,51}$ Most recently, Yanez et al. used Metropolis Monte Carlo methods to generate lignin topologies that satisfy measured molecular weight, monomer, and bond distributions. ${ }^{52,53}$ Leveraging these topologies, Broadbelt and coworkers have gained an improved understanding of lignin pyrolysis through the use of kinetic Monte Carlo methods. ${ }^{54}$ Further building upon this work, Vermaas et al. developed methods to convert the topological libraries produced by Yanez et al. to structural libraries resembling those of Jurasek, which simulate the three-dimensional structure of lignin within cell-wall-like environments. ${ }^{55}$ Parijs et al. were among the first to utilize kinetic Monte Carlo methods for predictive lignin simulations, but again their model was parameterized by information obtained from synthetic dehydrogenation polymers (DHPs). ${ }^{56}$ While the above methods are parameterized primarily by experimental data representing a population of macromolecules that may have changed during the extraction process, an alternative approach would be to parameterize models with data from ab initio predictions. Several prior density functional theory (DFT) calculations have investigated monolignol coupling focused on the thermodynamics of bond formation. ${ }^{57-61}$ If kinetics are invoked at all, it is only in the control of $\beta-O-4$ reactions, as thermodynamics alone cannot explain their qualitative frequency. ${ }^{62-64}$ Recently, we demonstrated that the full suite of radical coupling activation energies qualitatively reproduce many experimentally observed lignin findings, ${ }^{65}$ motivating the 
parameterization of stochastic models with these energies for deeper, more quantitative, understanding of lignin structure. To date, no lignification model currently exists that: (1) can be readily extended to newly-discovered monolignols, (2) is compatible with parameters derived from ab initio calculations that can be refined as the accuracy of such calculations improve, and (3) is easily accessible to the diverse community of chemists, engineers, and biologists focused on lignin utilization.

Here, we introduce LIGNIN-KMC, ${ }^{66}$ an open-source toolkit for the simulation of lignification that combines the

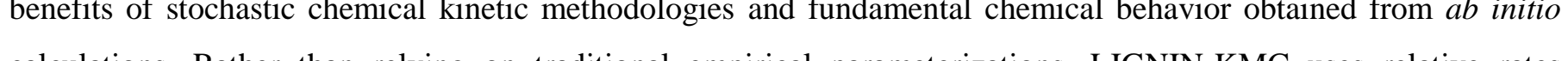
calculations. Rather than relying on traditional empirical parameterizations, LIGNIN-KMC uses relative rates estimated from the Eyring equation and transition state energetics calculated at the M06-2X/def2-TZVP level of theory. ${ }^{65}$ By using this hybrid strategy, we achieve monomer resolution within the polymer while incurring a lower computational cost as compared to ab initio simulations of the lignification process in a solvent. Close agreement between the simulated outputs and experimentally measured structural properties of lignins with typical hardwood and softwood compositions illustrates the predictive capability of the model. We then apply the LIGNIN-KMC method to investigate the impact of $\mathrm{S} / \mathrm{G}$ ratio on depolymerization monomer yields and to explore in detail the importance of monolignol addition rate as a parameter in controlling the resulting lignin chemistry in planta. With minimal additions, we extend the model to simulate the formation of homopolymers of caffeyl alcohol (C-unit) and accurately predict their bond formation tendencies. With the source code publicly available on GitHub, ${ }^{66}$ this toolkit can be readily used and built upon by researchers in areas focusing on lignin, ranging from plant biology and genetic engineering to catalytic depolymerization and renewable polymers derived from lignin, to gain deeper understanding of the lignification process and the implications of lignin structure on valorization strategies.

\section{Results and Discussion}

\subsection{Model Development}

LIGNIN-KMC relies on four main concepts: (1) A sparse matrix-based data structure to represent lignin in silico, (2) generation of possible reactions between lignin fragments, (3) computation of transition probabilities between two states, and (4) repetition of kinetic Monte Carlo simulations to obtain statistical distributions from which macroscopic properties can be measured.

\subsubsection{Data Structure}

A graph structure representing the chemical connectivity of lignin allows straightforward mapping of lignification into a computational framework. This graph contains $N$ nodes representing the simulated monomers, connected by edges whose weights signify the types of bonds connecting them. Figure 2(a) shows the numbering and types of monolignols that constitute lignin., ${ }^{1,2}$ 
a.

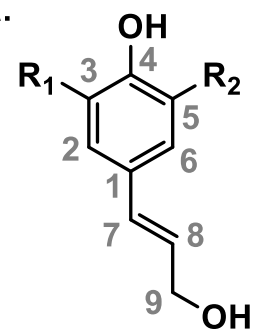

b.

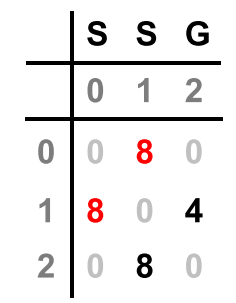

c.

Figure 2: Data structure used for lignin topology throughout the kinetic Monte Carlo simulations. The generic monolignols shown in (a) are sinapyl alcohol ( $S$-unit, $R_{1}=$ OMe, $R_{2}=$ OMe), coniferyl alcohol $\left(G\right.$-unit, $\left.R_{I}=O M e, R_{2}=H\right)$, p-coumaryl alcohol $(H$ unit, $\left.R_{1}=H, R_{2}=H\right)$, and caffeyl alcohol $\left(C\right.$-unit, $\left.R_{1}=H, R_{2}=O H\right)$. The adjacency matrix shown in $(b)$ contains two bonds, one $\beta$ - $\beta$ (highlighted in red) between monomers 0 and 1 , and one $\beta-O-4$ (highlighted in black) between monomers 1 and 2 . The chemical structure of a lignin fragment with this adjacency matrix is shown in $(c)$ with the same color highlighting, where the colored bonds represent the named bonds that are formed and the highlighted boxes show bonds around the formed bond that have changed as a consequence.

The $\beta-O-4, \beta-5, \beta-\beta, \beta-1, \alpha-O-4,5-5$, and 5-O-4 linkages that occur with measurable frequency in extracted lignins are easily stored in the adjacency matrix, shown by example in Figure 2(b). This matrix, A, corresponds to the fragment shown in Figure 2(c) and contains two bonds: red $\beta$ - $\beta$ between S-units 0 and $1, A_{01}=A_{10}=8$, and black $\beta$ - $O$ - 4 between S-unit 1 and G-unit $2, A_{12}=4, A_{21}=8$. The effects of rapid rearrangements accompanying the aforementioned bonds are highlighted by colored boxes in Figure 2(c). As compared to the string-based representations of lignin, ${ }^{56}$ the sparse adjacency matrix based graph structure provides a more compact, flexible, and efficient way to store the same information.

\subsubsection{Possible Simulation Events}

Oxidized fragments are known to react from the $\beta, 1,5$, and 4 positions with any other nearby oxidized fragments at these same positions to produce the observed $\beta-O-4, \beta-5, \beta-\beta, \beta-1, \alpha-O-4,5-5$, and 5-O-4 linkages. ${ }^{3,67}$ However, by allowing any monomer to react with any other monomer, the number of possible events grows rapidly with the number of monomers in the simulation. To avoid this, previous models have restricted the number of possible reactions by forcing reactions to occur only between monomers and a growing chain ${ }^{49}$ or by limiting the number of reactive sites within the monomers. ${ }^{56}$ Rather than following this approach, in LIGNIN-KMC, we allow all possible active sites on the monolignols to react with growing chains of any size to replicate the lignification process as it occurs within the cell walls of a plant with high fidelity. As such, we stipulate that only oxidized species may react with other oxidized species. We choose to neglect possible radical propagation reactions between a single oxidized species and a non- 
oxidized counterpart as these are predicted to incur large energetic penalties as compared to radical coupling. ${ }^{62}$ After the radical coupling, tautomerizations of all bonds other than $\beta-O-4$ were assumed to be instantaneous and irreversible, as none of these intermediate products are observed experimentally. ${ }^{1,2}$ For the $\beta-O-4$ bond, however, we explicitly considered the difference in barriers leading from the unstable quinone methide intermediate to (1) the $\beta$-O-4 linkage by hydration, and (2) the $\alpha-O-4$ bond by chain propagation.

In addition to chemical reaction rates, the rate of monolignol transport to the cell wall has been previously predicted to strongly influence lignin polymerization by altering instantaneous monolignol concentrations. ${ }^{56}$ To allow LIGNINKMC to dynamically add monolignols to the simulation, the adjacency matrix may grow in time. By combining the flexibility of the data structure with the extensibility of the event definitions, it is straightforward to incorporate new lignin understanding into this model structure.

\subsubsection{Transition Probabilities}

To accurately represent monomer coupling chemistry, we incorporated intrinsic chemical reaction rates associated with oxidation and coupling into the model. The coupling rates were approximated by applying the Eyring equation to energy barriers obtained from density functional theory (DFT) calculations (Table S1) ${ }^{65}$ for the reactions of monomer and dimer couplings to form $\beta-O-4, \beta-5, \beta-\beta, \beta-1, \alpha-O-4,5-5$, and $5-O-4$ bonds. Similarly, oxidation rates were approximated by calculating a radical transfer activation barrier with an $\mathrm{H}$-unit. Although DFT calculations are known to be error prone for absolute rate predictions, the relative rates, which benefit from error cancellation, ${ }^{61,68}$ dictate the probabilities of reaction. Equation (1) shows the rate calculation, where $h_{j}(\mathbf{X})$ is the rate of event $j$ in state $\mathbf{X}, \Delta G_{j}^{*}(\mathbf{X})$ is the difference between reactant and transition state energy obtained from DFT calculations, $k_{B}$ is the Boltzmann constant, $T$ the absolute temperature of the reaction $(T=298.15 \mathrm{~K}$ for all results presented here $), N$ is the number of monomers in the simulation, $n$ is the molecularity of the reaction, and $h$ is Planck's constant. Because each monomer is treated as an individual species, the concentration dependence of these rates is handled implicitly.

$$
h_{j}(\mathbf{X})=\frac{k_{B} T}{h N^{n}} \exp \left(-\frac{\Delta G_{j}^{\ddagger}(\mathbf{X})}{k_{B} T}\right)
$$

We treat the rate of monolignol transport as a parameter in lignification control. Previous models' reliance on experimental data to obtain transition probabilities only allows for regressive uses. However, based on new data available through DFT calculations ${ }^{65}$ we can obtain transition probabilities in a manner that supports predictive calculations. Although significant improvements have been realized in these DFT calculations, further insights could be offered by obtaining reaction rates from ab initio molecular dynamics-based simulations that can explicitly account for solvation or confinement effects during polymerization. We use the Gillespie algorithm, ${ }^{69,70}$ a well-known methodology for simulating chain polymerization reactions, ${ }^{71-73}$ to perform our stochastic simulation of the lignification process. Each iteration of the algorithm updates the state by drawing the time between events (stationary 
156 time) from an exponential distribution and selecting an event based on the weights determined through Equation (1).

157 In the representation of the Gillespie algorithm shown in Figure 3(a), $\mathbf{X}_{i}$ is the simulation state and $t_{i}$ is the simulation

158 time at iteration $i, R$ is the net rate of all possible events in the current state, $M$ is the number of events at the current

159 state, $u_{1}$ and $u_{2}$ are pseudo-random numbers on the uniform distribution, $\tau$ is the time that passes between the occurrence

160 of individual events, $j$ ' is the index of the specific event that will occur, and $f_{j}$, is the specific function that updates the

161 state to execute event $j$ '. For the mechanics of the implementation, see the source code. ${ }^{66}$

162 Figure 3(b) shows a schematic of the time evolution of the algorithm for a simple case of 3 S-units. The simulation

163 begins by considering neutral, disconnected monomers. The only possible events are the oxidations of the three

164 monomers. The rates of these events are normalized by the sum-total rate, stacked onto a number-line between 0 and

1651 , and compared to $u_{2}$ to perform the event selection. In the first iteration, the generated random numbers are $\mathbf{u}=$ $166[0.1791,0.6138]^{\mathrm{T}}$, which advances the simulation time to 1.72 and elects to oxidize the grey monomer. At the next 167 iteration, only the blue and the red monomers can be oxidized, and the choice of $\mathbf{u}=[0.3789,0.1012]^{\mathrm{T}}$ oxidizes the 168 blue monomer while advancing the time to 3.19. Now that there are two oxidized monomers, bonds could form between 169 them, or the red monomer could be oxidized. For the sake of this demonstration, $\mathbf{u}=[0.1341,0.9772]^{\mathrm{T}}$, causing the 170 bond to be formed between the two oxidized monomers. Based on the specific implementation, we avoid regeneration 171 of events that have already been accounted for, limiting the scaling to $O\left(N^{2.54}\right)$, as shown in Figure $\mathrm{S} 1$. To ease the 172 adaptation of these simulation results, we have also developed a method of converting the computational graph 173 structure into a molfile format for visual output and analysis, which is described in detail in the supporting information. 174 In addition, conversion tools have also been implemented to interface with other lignin analysis and modeling tools, 175 such as LigninBuilder ${ }^{55}$ and NetworkX. ${ }^{74}$ 
a.
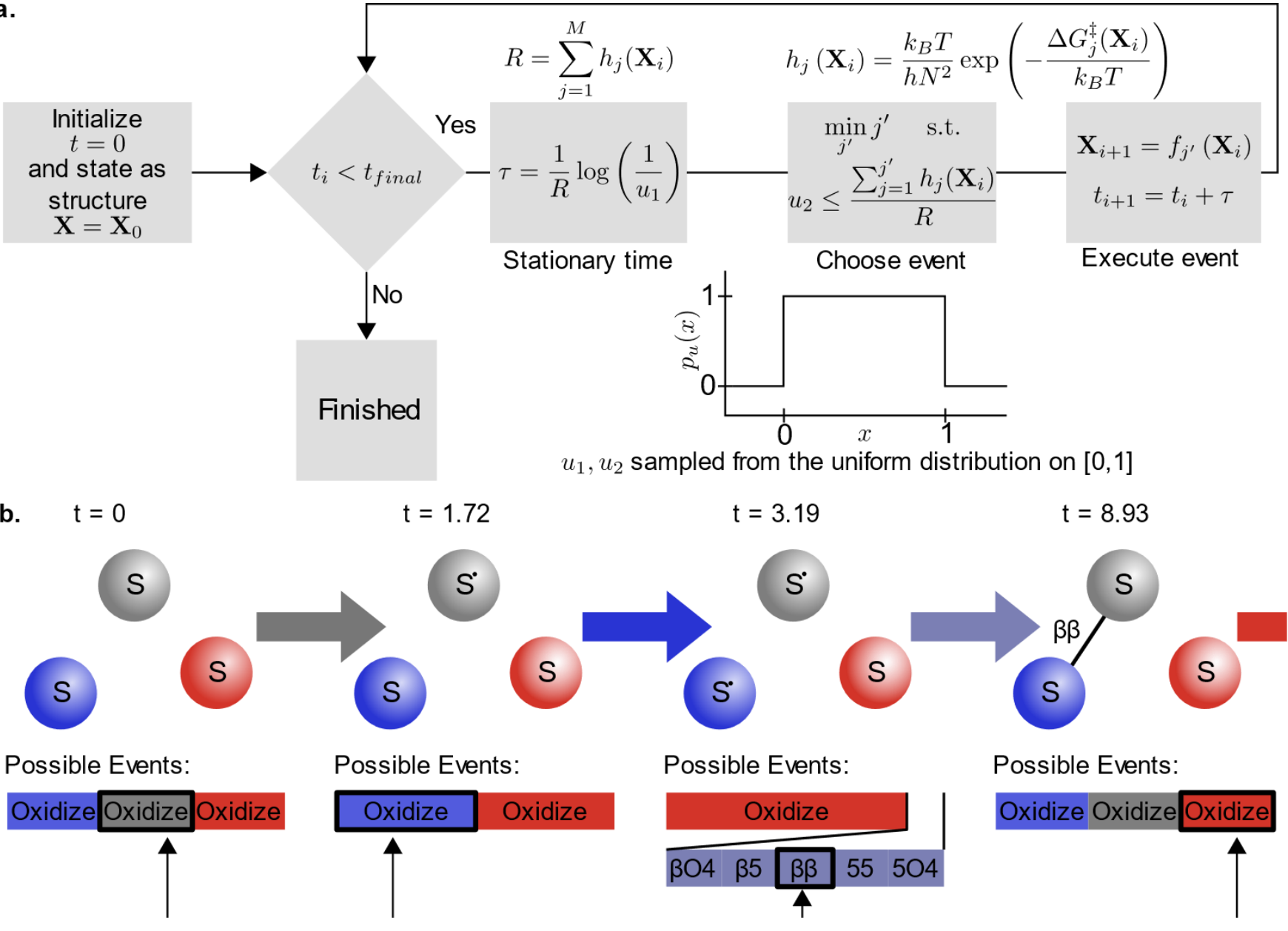

Figure 3: (a) The logic flow of the Gillespie algorithm used to perform the stochastic simulation of lignification. The state $\mathbf{X}$ is represented by the graph previously described. (b) Schematic evolution of a lignin state using the Gillespie algorithm. In this schematic, three $S$-units in their natural state are initialized in the simulation at $t=0$. Each frame of the schematic shows the possible events stacked onto a line normalized between 0 and 1 , where the length of the individual bar is proportional to the rate of the event listed. The arrow below this number line represents a random number selection that determines which event of the listed possibilities will be performed.

\subsubsection{Sampling Process}

As individual simulations are inherently stochastic by design, we rely on a method of repeated simulations and aggregated properties, as shown in Figure 4. The most important properties that we examine are the fractional bond contents and theoretical depolymerization monomer yields, as could be obtained through reductive catalytic fractionation (RCF). The bond contents can be obtained directly from the adjacency matrix, and the monomer yields are obtained by breaking all $\mathrm{C}-\mathrm{O}$ bonds formed during lignification, as RCF depolymerization processes do, ${ }^{75}$ while leaving $\mathrm{C}-\mathrm{C}$ bonds intact. Once the $\mathrm{C}-\mathrm{O}$ bonds have been removed from the adjacency matrix, we use depth-first search techniques described in the supporting information to identify and size the resulting lignin fragments. We envision that in addition to the use cases presented in the introduction, analytical chemists that study lignin will find tools based on LIGNIN-KMC to be an invaluable starting place for understanding and studying higher molecular weight fragments. 


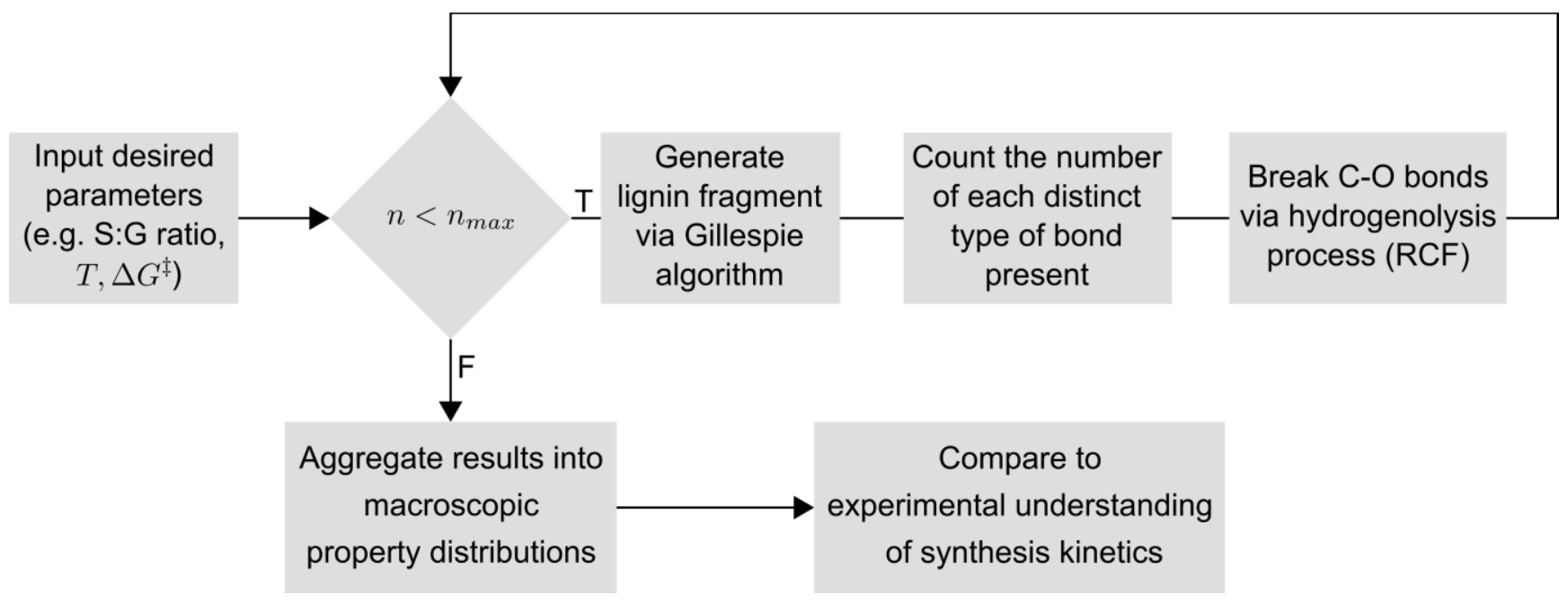

Figure 4: Schematic visualization of sampling methodology used for generation of macroscopic property descriptors of lignin generated in silico. Bonds are counted directly from the adjacency matrix, while $C$-O bonds formed during synthesis are broken instantaneously in this simulation to attempt to observe the theoretical monomer yields obtained from a reductive catalytic fractionation (RCF) process.

\subsection{Model Validation}

In section 2.1, we described the LIGNIN-KMC model of lignification processes; here we validate the model and study important open questions in the literature. More specifically, we first investigate the polymerization of lignin composed of varying amounts of S- and G-monomers, with these monomers added to the simulation at varying rates. Through this study, we hope to understand the localization of bonds that are present in the final polymer and the impact that kinetics and transport have on final structure. Second, we study the homo-polymerization of caffeyl alcohol monomers, as these types of polymers have previously been difficult to rationalize computationally.

\subsubsection{Analysis of $S / G$ Lignins}

Recent studies on the genetic engineering of lignins as feedstocks ${ }^{35-38}$ for biorefineries have focused on increasing the ratio of S-units to G-units within the polymer as this is hypothesized to increase the fraction of $\beta-O-4$ bonds in the final structure, which should subsequently increase the monomer yield from RCF. ${ }^{76}$ To date, studies on the RCF of different biomass species have identified a clear correlation between S/G ratio and depolymerization yield. ${ }^{21}$ Genetically modified poplars have been found to be similarly correlated, where high S-content results in high monomer yields. ${ }^{38,77-}$ ${ }^{79}$ However, native variants of the same plant species were not similarly correlated, calling into question previous assumptions about how lignin forms from its constituent monomers. ${ }^{80}$ Anderson et al. further investigated this effect by studying the distribution of dimers obtained from RCF of different poplars, showing that the S-units were more likely to form C-C dimers. ${ }^{80}$ Accordingly, a deeper understanding of the lignification process would enable rational tuning of the control mechanisms that plants use to defend against biological degradation and would help differentiate between competing hypotheses formulated to explain the observed yields in RCF processing. 
217 Based on its flexibility, our model can fully evaluate the two-dimensional space of monomer composition and transport 218 rate to investigate these hypotheses. The discrepancies amongst the prior literature on the impact of S/G ratio suggest 219 the existence of tunable mechanisms for controlling bond frequency and maximum monomer yields. To test the 220 influence of S/G ratio within the model framework, we systematically varied the $\mathrm{S}$ to $\mathrm{G}$ ratio between 0.1 and 10 at 221 constant monolignol transport rates, as shown in Figure 5. The monolignol transport rates that are investigated here 222 span 1 monomer per second to $10^{8}$ monomers per second added to the simulation. This range of transport rates was 223 selected to approach the typical bond formation rates, which for the computed $\beta-O-4$ energetics is $1.5 \times 10^{8}$ reactions 224 per second, such that both mass transport and kinetically limited regimes could be explored. In this study, the transport 225 rate relative to the rates of bond formation events are more relevant than the absolute transport rates. The lowest selected 226 transport rate of 1 monomer per second for individual chains is likely in the correct order of magnitude range for 227 passive monolignol transport. The rate of monolignol transport from the entire cell can be estimated at about $10^{8}$ 228 monomers per second, ${ }^{81}$ which would be reduced significantly by spatial restrictions on lignin coupling. At the high 229 end, the transport rates correspond to in vitro processes or the rapid release of lignin stored within the vacuole..$^{82}$ Given 230 the inherent uncertainty around the native addition rate, all of the chosen transport rates may be justified under certain 231 conditions. Thus, rather than focus on any particular case, we parametrically sweep the transport rate and focus on the 232 general trends that emerge in the bond distribution. 

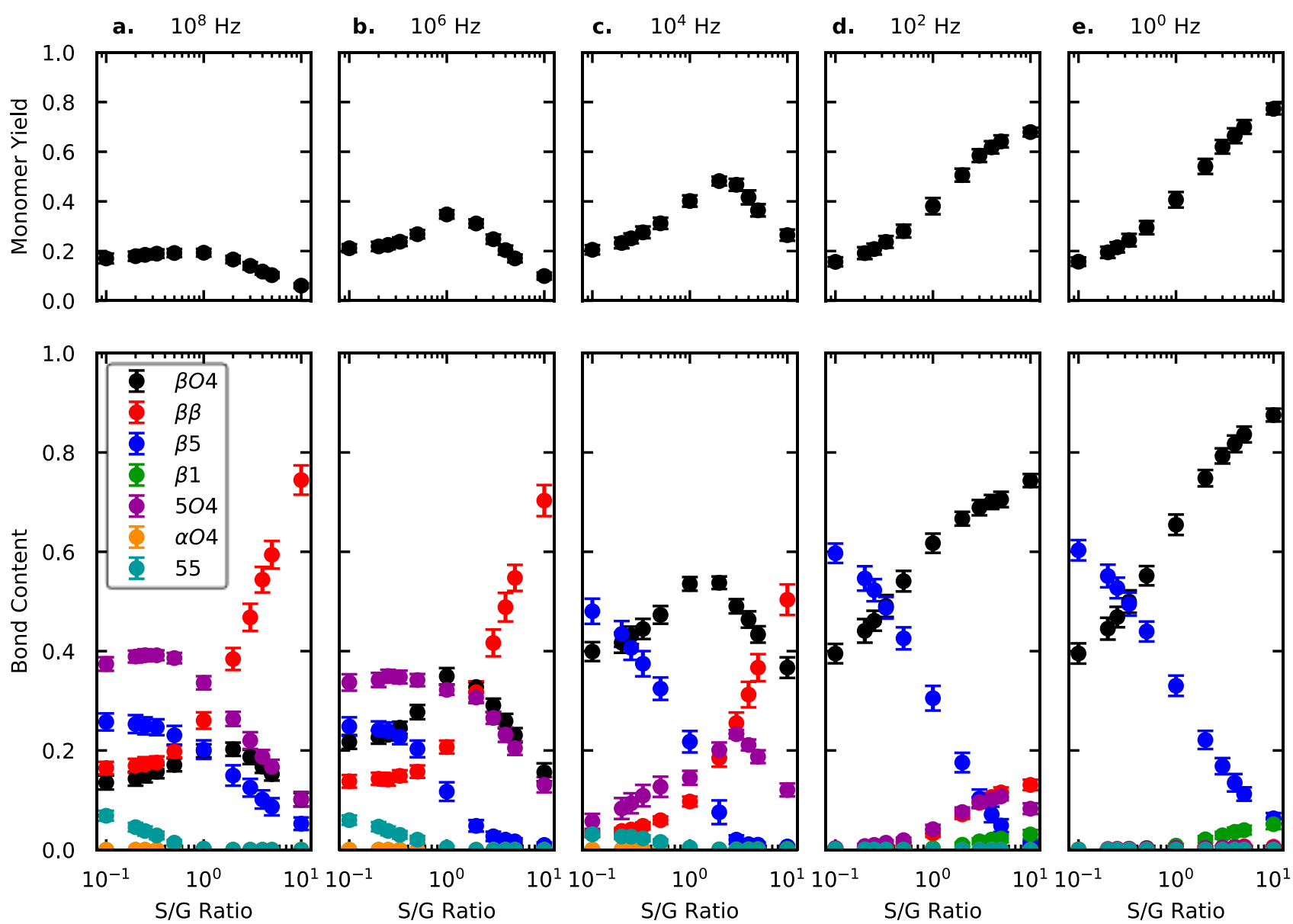

Figure 5: Bond and monomer properties measured from lignin generated in silico under different polymerization conditions where (top) shows the yields of monomers obtained after degradation by ether cleavage treatments and (bottom) shows the fraction of bonds identified in the lignin fragments before hydrogenolysis. (a) The effect of $S / G$ ratio on lignin properties under conditions of high monolignol concentration (e.g., batch polymerization), (b-d) the effect of monomer addition rate on lignin properties with a fixed $S / G$ ratio of 1 , and $(e)$ the effect of $S / G$ ratio on lignin properties when monolignols are limiting (e.g., continuous polymerization). In each panel, the respective transport rate is displayed at top in units of monomers per second.

In Figure 5 there are clear trends in the theoretical monomer yields (top panel) and bond frequencies (bottom panels) as both the $\mathrm{S} / \mathrm{G}$ ratio and monolignol transport rates are varied. Broadly speaking, as the monolignol transport rate increases, there is a larger maximum $\beta-\beta$ bond content and lower maximum $\beta-O-4$ and $\beta-5$ bond content. These changes are directly reflected by lower maximum monomer yields, due to the decreased prevalence for $\mathrm{C}-\mathrm{O}$ bonds.

244 The monolignol transport rate has been explored experimentally in the literature through the synthesis of DHP lignins via batch (Zulauf-type) and slow-feed (Zutropf-type) processes. The data observed under conditions mimicking Zulauf DHP experiments, where monomers are added quickly, are shown in Figure 5(a). The trend of decreasing monomer yield with increasing S-content appears contrary to most observations and intuition. However, in this case, LIGNIN$\mathrm{KMC}$ predicts a higher frequency of $\beta$ - $\beta$ linkages created between S-units that terminate the polymer growth, wereas competing linkages at the 5- position are common in high-G-lignin. Most reports of Zulauf polymerization experiments 
suggest the presence of many low molecular weight fragments, with the occurrence of primarily $\beta$ - $\beta$ bonds between $S$ units, ${ }^{83}$ and $\beta-5$ bonds between G-units. ${ }^{84}$ Interestingly, we also see a large fraction of 5-O-4 connections between high G-content lignins, but these tend to form between larger fragments after the formation of $\beta-5$ bonds (Figures S3-5). To more closely match observed yields, we attempted simulating lignification under conditions that may more closely resemble those in planta, e.g. Zutropf experiments in vitro. Under these slow monomer addition conditions, shown in Figure 5(e), increasing S-content drastically increases $\beta-O-4$ content, which subsequently increases the depolymerization monomer yield (Figure S6), as previously predicted. ${ }^{76,85}$ This appears to match what has been observed with transgenic lignins, ${ }^{38,77}$ and those generated during Zutropf DHP experiments. ${ }^{86-88}$ In addition, under these conditions, the bond distributions that we simulate match closely what has been reported previously. ${ }^{9,67}$ At an $S / G$ ratio of 1 and transport rate of 1 monomer per second, we observe a $\beta-O-4$ bond frequency near $65 \%$, while typical reported values are between $50-62 \% .9,67$ Similarly, we observe $\beta-\beta$ bond contents of near $1 \%$ while reported values are usually between $2-12 \% .{ }^{9,67}$ The largest deviations that we see exist in $\beta-5$ and $\beta-1$ bonds, where we expect $3-11 \%$ but observe $33 \%$ and expect $1-7 \%$ but observe $1 \%$ respectively. ${ }^{9,67}$ The relatively high frequency of $\beta-5$ bonds is more similar to what has been observed in DHP lignins,${ }^{84}$ but the differences between the in vitro polymers and native lignins remains unclear. Based on the stark contrast between these two limiting cases, we found it important to investigate some of the intermediate transport rate behavior.

The data corresponding to these intermediate transport regimes are shown in Figure 5(b-d). These data show the gradual changes in predicted bond content and depolymerization yields as a function of transport rate. Interestingly, it is possible to observe extrema in the monomer yields at moderate $S / G$ ratios, which can be explained by a tradeoff between high $\beta$ - $\beta$ bond content between S-units and high $\beta$-5 bond content between G-units. Perhaps unsurprisingly given the behavior in the limiting cases, at intermediate monolignol transport rates, regions of relative invariance appear in the monomer yield as the $\mathrm{S} / \mathrm{G}$ ratio changes. The existence of such plateaus could help explain why monomer yields do not appear to change as $\mathrm{S} / \mathrm{G}$ ratio changes in wild type lignins. ${ }^{80}$

\subsubsection{Analysis of Non-Traditional Lignins}

Recent discoveries of lignins composed of building blocks beyond traditional S- and G-monomers have inspired a number of different applications. ${ }^{39,44,89-91}$ These monomers are still typically phenylalanine-derived species but are under-methylated as compared to their S-, G-, and H-counterparts. Homopolymers of caffeyl alcohol (C-lignin) hold particular promise as a feedstock for fuel and chemical production due to the near exclusive presence of interunit benzodioxane linkages, a specific type of $\beta-O-4$ linkage that can easily be cleaved.$^{89,90,92,93}$ Based on this discovery, researchers have sought to understand the thermodynamic origin of the selectivity of benzodioxane linkages compared to a hydrated $\beta-O-4$ linkage. ${ }^{90}$ In addition, recent efforts have been made to take advantage of the well-defined ether linkage structure by using C-lignins in RCF treatments, where, despite the high theoretical yields, catalyst deactivation 
282 limits the actual yields. ${ }^{92,93}$ Therefore, in the present model, it is more informative to compare the results of bond 283 contents than those of monomer yields as would be possible for S/G-lignin.

284 The tunability of the model enables simulation of these novel lignins by defining new monomer types and updating the 285 set of transition state energies obtained from kinetic DFT calculations (Table S2). Notably, with those two changes, 286 the same methodology employed in the previous section and knowledge gained for modeling S/G lignins can be applied 287 to guide the exploration of this polymer. Specifically, C-lignin was synthesized in silico using a transport rate of $1 \mathrm{C}$ 288 units per second, as identified as being sufficiently slow in the S/G-lignin above. The distribution for the simulations 289 run is shown in Figure 6. Despite the lower predicted activation barrier of $\beta-5$ bond formation for the coupling of two 290 monomers, our calculations show the resulting lignin is highly enriched in $\beta-O-4$ content, matching the native lignin 291 found in seed coats as determined by experimentation. ${ }^{89}$ This counter-intuitive result can again be understood by 292 comparing the energetics of monomer-monomer additions with monomer-chain additions (Table S2). Thus, with 293 minimal adaptation, we are able to accurately describe the structure of a new experimentally characterized lignin type. 294 We anticipate that, as new lignin forms are discovered, or additional parameters of interest are identified, this model 295 can be easily adjusted to incorporate this new behavior.

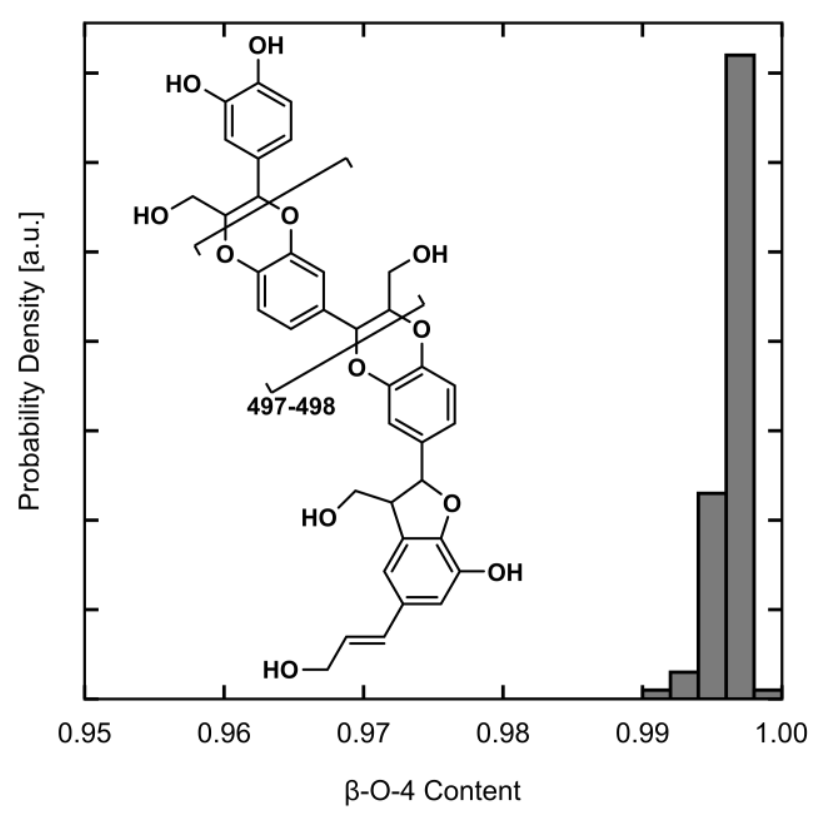

Figure 6: $\beta$-O-4 bond content distribution for the prediction of $C$-lignin generation. For these simulations, monolignols were added at a rate of $1 \mathrm{~Hz}$, starting with 2 monomers, with a maximum of 500 monomers included.

\section{Conclusions}

300 In this work, we demonstrated the general framework and broad applicability of the LIGNIN-KMC toolkit for 301 simulating lignification using both traditional S/G-lignin and the recently discovered C-lignin. Through these 302 applications, we have garnered better understanding of the chemistry that occurs during lignification and have 
illustrated the impact of monolignol supply rate on lignin structure. Specifically, we have shown, for the first time, the behavior of lignification chemistry where transport processes occur on timescales similar to the bond formation kinetics. Through this study, regions of relative invariance of depolymerization yields with changing S/G ratio were identified that could have significant implications for plant biologists attempting to create "designer" lignins. Further, our studies allow us to understand the structure of these S/G lignins that will eventually be used by both the catalytic depolymerization scientists and polymer scientists, providing an improved starting point for their optimizations and engineering. We have also shown that, with minimal adaptations, this model can accurately simulate the formation of the C-lignin polymer, which has previously not been attempted. From this point, the LIGNIN-KMC model can be further used to explore different biosynthetic parameters of interest to help provide a better starting point for more realistic models of designer lignins for use in detailed computational studies. We note that this model is currently unable to explore the effects of spatial orientation or confinement of lignin fragments within the cell wall, which we hypothesize could also have significant impact on the final lignin structure. To that end, LIGNIN-KMC serves as a foundation for future studies and extensions that incorporate these types of effects, including time-dependent monomer delivery rates, leading to a more comprehensive, molecular understanding of the lignification process.

\section{Acknowledgements}

The work performed was supported by the National Science Foundation, CBET Award No 1454299, and the Center for Bioenergy Innovation a U.S. Department of Energy Research Center supported by the Office of Biological and Environmental Research in the DOE Office of Science. This work used the Extreme Science and Engineering Discovery Environment (XSEDE) Comet at the San Diego Super Computer Cluster through allocations TGCTS180027, which is supported by National Science Foundation grant number ACI-1548562; and TG-MCB090159 which is supported by National Science Foundation grant number ACI-1053575. MJO gratefully acknowledges the National Science Foundation Graduate Research Fellowship Program for financial support. This material is based upon work supported by the National Science Foundation Graduate Research Fellowship under Grant No. 1122374. Any opinion, findings, and conclusions or recommendations expressed in this material are those of the authors and do not necessarily reflect the views of the National Science Foundation. The authors thank Connor Coley and McLain Leonard for insightful discussion and constructive feedback.

\section{References}

(1) Boerjan, W.; Ralph, J.; Baucher, M. Lignin Biosynthesis. Annu. Rev. Plant Biol. 2003, 54 (1), 519-546.

(2) Ralph, J.; Lundquist, K.; Brunow, G.; Lu, F.; Kim, H.; Schatz, P. F.; Marita, J. M.; Hatfield, R. D.; Ralph, S. A.; Christensen, J. H.; et al. Lignins: Natural Polymers from Oxidative Coupling of 4-HydroxyphenylPropanoids. Phytochem. Rev. 2004, 3, 29-60.

(3) Vanholme, R.; Demedts, B.; Morreel, K.; Ralph, J.; Boerjan, W. Lignin Biosynthesis and Structure. Plant Physiol. 2010, 153 (3), 895-905.

(4) Zhao, Q.; Dixon, R. A. Transcriptional Networks for Lignin Biosynthesis: More Complex than We Thought? Trends Plant Sci. 2011, 16 (4), 227-233. 
338

339

340

341

342

343

344

345

346

347

348

349

350

351

352

353

354

355

356

357

358

359

360

361

362

363

364

365

366

367

368

369

370

371

372

373

374

375

376

377

378

379

380

381

382

383

384

385

386

387

(5) Weng, J.-K.; Chapple, C. The Origin and Evolution of Lignin Biosynthesis. New Phytol. 2010, 187 (2), 273285.

(6) Huber, G. W.; Iborra, S.; Corma, A. Synthesis of Transportation Fuels from Biomass: Chemistry, Catalysts, and Engineering. Chem. Rev. 2006, 106 (9), 4044-4098.

(7) Ragauskas, A. J.; Williams, C. K.; Davison, B. H.; Britovsek, G.; Cairney, J.; Eckert, C. A.; Frederick, W. J.; Hallett, J. P.; Leak, D. J.; Liotta, C. L.; et al. The Path Forward for Biofuels and Biomaterials. Science 2006, 311 (5760), 484-489.

(8) Ragauskas, A. J.; Beckham, G. T.; Biddy, M. J.; Chandra, R.; Chen, F.; Davis, M. F.; Davison, B. H.; Dixon, R. A.; Gilna, P.; Keller, M.; et al. Lignin Valorization: Improving Lignin Processing in the Biorefinery. Science 2014, 344 (6185), 1246843.

(9) Rinaldi, R.; Jastrzebski, R.; Clough, M. T.; Ralph, J.; Kennema, M.; Bruijnincx, P. C. A.; Weckhuysen, B. M. Paving the Way for Lignin Valorisation: Recent Advances in Bioengineering, Biorefining and Catalysis. Angew. Chem. Int. Ed. 2016, 55 (29), 8164-8215.

(10) Schutyser, W.; Renders, T.; Bosch, S. V. den; Koelewijn, S.-F.; Beckham, G. T.; Sels, B. F. Chemicals from Lignin: An Interplay of Lignocellulose Fractionation, Depolymerisation, and Upgrading. Chem. Soc. Rev. 2018, 47 (3), 852-908.

(11) Davis, R.; Tao, L.; Tan, E. C. D.; Biddy, M. J.; Beckham, G. T.; Scarlata, C.; Jacobson, J.; Cafferty, K.; Ross, J.; Lukas, J.; et al. Process Design and Economics for the Conversion of Lignocellulosic Biomass to Hydrocarbons: Dilute-Acid and Enzymatic Deconstruction of Biomass to Sugars and Biological Conversion of Sugars to Hydrocarbons; NREL/TP-5100-60223; National Renewable Energy Lab. (NREL), Golden, CO (United States), 2013.

(12) Su, Y.; Brown, H. M.; Huang, X.; Zhou, X.; Amonette, J. E.; Zhang, Z. C. Single-Step Conversion of Cellulose to 5-Hydroxymethylfurfural (HMF), a Versatile Platform Chemical. Appl. Catal. Gen. 2009, 361 (1), 117-122.

(13) Luterbacher, J. S.; Alonso, D. M.; Rand, J. M.; Questell-Santiago, Y. M.; Yeap, J. H.; Pfleger, B. F.; Dumesic, J. A. Solvent-Enabled Nonenyzmatic Sugar Production from Biomass for Chemical and Biological Upgrading. ChemSusChem 2015, 8 (8), 1317-1322.

(14) Luterbacher, J. S.; Rand, J. M.; Alonso, D. M.; Han, J.; Youngquist, J. T.; Maravelias, C. T.; Pfleger, B. F.; Dumesic, J. A. Nonenzymatic Sugar Production from Biomass Using Biomass-Derived $\gamma$-Valerolactone. Science 2014, 343 (6168), 277-280.

(15) Fatih Demirbas, M. Biorefineries for Biofuel Upgrading: A Critical Review. Appl. Energy 2009, 86, S151S161.

(16) Martin Alonso, D.; G. Wettstein, S.; A. Dumesic, J. Bimetallic Catalysts for Upgrading of Biomass to Fuels and Chemicals. Chem. Soc. Rev. 2012, 41 (24), 8075-8098.

(17) Van de Vyver, S.; Geboers, J.; Jacobs, P. A.; Sels, B. F. Recent Advances in the Catalytic Conversion of Cellulose. ChemCatChem 2011, 3 (1), 82-94.

(18) Zhang, Y.-H. P.; Lynd, L. R. Toward an Aggregated Understanding of Enzymatic Hydrolysis of Cellulose: Noncomplexed Cellulase Systems. Biotechnol. Bioeng. 2004, 88 (7), 797-824.

(19) Alvira, P.; Tomás-Pejó, E.; Ballesteros, M.; Negro, M. J. Pretreatment Technologies for an Efficient Bioethanol Production Process Based on Enzymatic Hydrolysis: A Review. Bioresour. Technol. 2010, 101 (13), 48514861.

(20) Langston, J. A.; Shaghasi, T.; Abbate, E.; Xu, F.; Vlasenko, E.; Sweeney, M. D. Oxidoreductive Cellulose Depolymerization by the Enzymes Cellobiose Dehydrogenase and Glycoside Hydrolase 61. Appl. Environ. Microbiol. 2011, 77 (19), 7007-7015.

(21) Bosch, S. V. den; Schutyser, W.; Vanholme, R.; Driessen, T.; Koelewijn, S.-F.; Renders, T.; Meester, B. D.; Huijgen, W. J. J.; Dehaen, W.; Courtin, C. M.; et al. Reductive Lignocellulose Fractionation into Soluble LigninDerived Phenolic Monomers and Dimers and Processable Carbohydrate Pulps. Energy Environ. Sci. 2015, 8 (6), 1748-1763.

(22) Bosch, S. V. den; Schutyser, W.; Koelewijn, S.-F.; Renders, T.; Courtin, C. M.; Sels, B. F. Tuning the Lignin Oil OH-Content with Ru and Pd Catalysts during Lignin Hydrogenolysis on Birch Wood. Chem. Commun. 2015, 51 (67), 13158-13161. 
(23) Anderson, E. M.; Katahira, R.; Reed, M.; Resch, M. G.; Karp, E. M.; Beckham, G. T.; Román-Leshkov, Y. Reductive Catalytic Fractionation of Corn Stover Lignin. ACS Sustain. Chem. Eng. 2016, 4 (12), 6940-6950.

(24) Ten, E.; Vermerris, W. Recent Developments in Polymers Derived from Industrial Lignin. J. Appl. Polym. Sci. 2015, 132 (24).

(25) Holmberg, A. L.; Nguyen, N. A.; Karavolias, M. G.; Reno, K. H.; Wool, R. P.; Epps, T. H. Softwood LigninBased Methacrylate Polymers with Tunable Thermal and Viscoelastic Properties. Macromolecules 2016, 49 (4), 1286-1295.

(26) Naseem, A.; Tabasum, S.; Zia, K. M.; Zuber, M.; Ali, M.; Noreen, A. Lignin-Derivatives Based Polymers, Blends and Composites: A Review. Int. J. Biol. Macromol. 2016, 93, 296-313.

(27) Brzonova, I.; Kozliak, E. I.; Andrianova, A. A.; LaVallie, A.; Kubátová, A.; Ji, Y. Production of Lignin Based Insoluble Polymers (Anionic Hydrogels) by C. Versicolor. Sci. Rep. 2017, 7 (1), 17507.

(28) Wang, S.; Shuai, L.; Saha, B.; Vlachos, D. G.; Epps, T. H. From Tree to Tape: Direct Synthesis of Pressure Sensitive Adhesives from Depolymerized Raw Lignocellulosic Biomass. ACS Cent. Sci. 2018, 4 (6), 701-708.

(29) Grossman, A.; Vermerris, W. Lignin-Based Polymers and Nanomaterials. Curr. Opin. Biotechnol. 2019, 56, $112-120$.

(30) Liitiä, T. M.; Maunu, S. L.; Hortling, B.; Toikka, M.; Kilpeläinen, I. Analysis of Technical Lignins by Twoand Three-Dimensional NMR Spectroscopy. J. Agric. Food Chem. 2003, 51 (8), 2136-2143.

(31) D Mansfield, S.; Kim, H.; Lu, F.; Ralph, J. Whole Plant Cell Wall Characterization Using 2D-NMR. Nat. Protoc. 2012, 7, 1579-1589.

(32) Sette, M.; Lange, H.; Crestini, C. Quantitative HSQC Analyses of Lignin: A Practical Comparison. Comput. Struct. Biotechnol. J. 2013, 6 (7), e201303016.

(33) Wen, J.-L.; Sun, S.-L.; Xue, B.-L.; Sun, R.-C. Recent Advances in Characterization of Lignin Polymer by Solution-State Nuclear Magnetic Resonance (NMR) Methodology. Materials 2013, 6 (1), 359-391.

(34) Ralph, J.; Landucci, L. L. NMR of Lignins. In Lignin and Lignans: Advances in Chemistry; CRC Press, 2016; pp 137-244.

(35) Fu, C.; Mielenz, J. R.; Xiao, X.; Ge, Y.; Hamilton, C. Y.; Rodriguez, M.; Chen, F.; Foston, M.; Ragauskas, A.; Bouton, J.; et al. Genetic Manipulation of Lignin Reduces Recalcitrance and Improves Ethanol Production from Switchgrass. Proc. Natl. Acad. Sci. 2011, 108 (9), 3803-3808.

(36) Hisano, H.; Nandakumar, R.; Wang, Z.-Y. Genetic Modification of Lignin Biosynthesis for Improved Biofuel Production. In Biofuels: Global Impact on Renewable Energy, Production Agriculture, and Technological Advancements; Tomes, D., Lakshmanan, P., Songstad, D., Eds.; Springer New York: New York, NY, 2011; pp 223-235.

(37) Li, X.; Weng, J.-K.; Chapple, C. Improvement of Biomass through Lignin Modification. Plant J. 2008, 54 (4), 569-581.

(38) Stewart, J. J.; Akiyama, T.; Chapple, C.; Ralph, J.; Mansfield, S. D. The Effects on Lignin Structure of Overexpression of Ferulate 5-Hydroxylase in Hybrid Poplar1. Plant Physiol. 2009, 150 (2), 621-635.

(39) Oyarce, P.; De Meester, B.; Fonseca, F.; de Vries, L.; Goeminne, G.; Pallidis, A.; De Rycke, R.; Tsuji, Y.; Li, Y.; Van den Bosch, S.; et al. Introducing Curcumin Biosynthesis in Arabidopsis Enhances Lignocellulosic Biomass Processing. Nat. Plants 2019, 5 (2), 225-237.

(40) Bonawitz, N. D.; Chapple, C. The Genetics of Lignin Biosynthesis: Connecting Genotype to Phenotype. Annu. Rev. Genet. 2010, 44 (1), 337-363.

(41) Liu, C.-J. Deciphering the Enigma of Lignification: Precursor Transport, Oxidation, and the Topochemistry of Lignin Assembly. Mol. Plant 2012, 5 (2), 304-317.

(42) Shi, R.; Sun, Y.-H.; Li, Q.; Heber, S.; Sederoff, R.; Chiang, V. L. Towards a Systems Approach for Lignin Biosynthesis in Populus Trichocarpa: Transcript Abundance and Specificity of the Monolignol Biosynthetic Genes. Plant Cell Physiol. 2010, 51 (1), 144-163.

(43) Moura, J. C. M. S.; Bonine, C. A. V.; Viana, J. D. O. F.; Dornelas, M. C.; Mazzafera, P. Abiotic and Biotic Stresses and Changes in the Lignin Content and Composition in Plants. J. Integr. Plant Biol. 2010, 52 (4), 360376.

(44) Ralph, J. Hydroxycinnamates in Lignification. Phytochem. Rev. 2010, 9 (1), 65-83. 
(45) Mottiar, Y.; Vanholme, R.; Boerjan, W.; Ralph, J.; Mansfield, S. D. Designer Lignins: Harnessing the Plasticity of Lignification. Curr. Opin. Biotechnol. 2016, 37, 190-200.

(46) Barros, J.; Serrani-Yarce, J. C.; Chen, F.; Baxter, D.; Venables, B. J.; Dixon, R. A. Role of Bifunctional Ammonia-Lyase in Grass Cell Wall Biosynthesis. Nat. Plants 2016, 2 (6), 16050.

(47) Liu, C.-J.; Miao, Y.-C.; Zhang, K.-W. Sequestration and Transport of Lignin Monomeric Precursors. Molecules 2011, 16 (1), 710-727.

(48) Hatfield, R.; Vermerris, W. Lignin Formation in Plants. The Dilemma of Linkage Specificity. Plant Physiol. 2001, 126 (4), 1351-1357.

(49) Glasser, W. G.; Glasser, H. R. Simulation of Reactions with Lignin by Computer (SIMREL). I. Polymerization of Coniferyl Alcohol Monomers. Macromolecules 1974, 7 (1), 17-27.

(50) Jurasek, L. Toward a 3-Dimensional Model of Lignin Structure. J. Pulp Pap. Sci. 1995, 21 (8), J274-J279.

(51) Jurasek, L. Molecular Modelling of Fibre Walls. J. Pulp Pap. Sci. 1998, 24 (7), 209-212.

(52) Yanez, A. J.; Li, W.; Mabon, R.; Broadbelt, L. J. A Stochastic Method to Generate Libraries of Structural Representations of Lignin. Energy Fuels 2016, 30 (7), 5835-5845.

(53) Dellon, L. D.; Yanez, A. J.; Li, W.; Mabon, R.; Broadbelt, L. J. Computational Generation of Lignin Libraries from Diverse Biomass Sources. Energy Fuels 2017, 31 (8), 8263-8274.

(54) Yanez, A. J.; Natarajan, P.; Li, W.; Mabon, R.; Broadbelt, L. J. Coupled Structural and Kinetic Model of Lignin Fast Pyrolysis. Energy Fuels 2018, 32 (2), 1822-1830.

(55) Vermaas, J. V.; Dellon, L. D.; Broadbelt, L. J.; Beckham, G. T.; Crowley, M. F. Automated Transformation of Lignin Topologies into Atomic Structures with LigninBuilder. ACS Sustain. Chem. Eng. 2018.

(56) Parijs, F. R. D. van; Morreel, K.; Ralph, J.; Boerjan, W.; Merks, R. M. H. Modeling Lignin Polymerization. I. Simulation Model of Dehydrogenation Polymers. Plant Physiol. 2010, 153 (3), 1332-1344.

(57) Watts, H. D.; Mohamed, M. N. A.; Kubicki, J. D. Evaluation of Potential Reaction Mechanisms Leading to the Formation of Coniferyl Alcohol $\alpha$-Linkages in Lignin: A Density Functional Theory Study. Phys. Chem. Chem. Phys. 2011, 13 (47), 20974-20985.

(58) Sangha, A. K.; Parks, J. M.; Standaert, R. F.; Ziebell, A.; Davis, M.; Smith, J. C. Radical Coupling Reactions in Lignin Synthesis: A Density Functional Theory Study. J. Phys. Chem. B 2012, 116 (16), 4760-4768.

(59) Beste, A.; Buchanan, A. C. Computational Study of Bond Dissociation Enthalpies for Lignin Model Compounds. Substituent Effects in Phenethyl Phenyl Ethers. J. Org. Chem. 2009, 74 (7), 2837-2841.

(60) Younker, J. M.; Beste, A.; Buchanan, A. C. Computational Study of Bond Dissociation Enthalpies for Substituted $\beta-O-4$ Lignin Model Compounds. Chemphyschem Eur. J. Chem. Phys. Phys. Chem. 2011, 12 (18), 3556-3565.

(61) Kim, S.; Chmely, S. C.; Nimlos, M. R.; Bomble, Y. J.; Foust, T. D.; Paton, R. S.; Beckham, G. T. Computational Study of Bond Dissociation Enthalpies for a Large Range of Native and Modified Lignins. J. Phys. Chem. Lett. 2011, 2 (22), 2846-2852.

(62) Durbeej, B.; Eriksson, L. A. Formation of $\beta$-O-4 Lignin Models -A Theoretical Study. Holzforschung 2005, 57 (5), 466-478.

(63) Shigematsu, M.; Kobayashi, T.; Taguchi, H.; Tanahashi, M. Transition State Leading to $\beta-\mathrm{O}^{\prime}$ Quinonemethide Intermediate of p-Coumaryl Alcohol Analyzed by Semi-Empirical Molecular Orbital Calculation. J. Wood Sci. 2006, 52 (2), 128-133.

(64) Sangha, A. K.; Davison, B. H.; Standaert, R. F.; Davis, M. F.; Smith, J. C.; Parks, J. M. Chemical Factors That Control Lignin Polymerization. J. Phys. Chem. B 2014, 118 (1), 164-170.

(65) Gani, T.; Orella, M.; Anderson, E.; Stone, M.; Brushett, F.; Beckham, G.; Román-Leshkov, Y. Quantum Mechanical Calculations Suggest That Lignin Polymerization Is Kinetically Controlled. 2018.

(66) Orella, M. Michaelorella/Lignin-Kmc.

(67) Dimmel, D. Overview. In Lignins and Lignans: Advances in Chemistry; CRC Press, 2016.

(68) Zhao, Y.; Truhlar, D. G. Density Functionals with Broad Applicability in Chemistry. Acc. Chem. Res. 2008, 41 (2), 157-167.

(69) Gillespie, D. T. A General Method for Numerically Simulating the Stochastic Time Evolution of Coupled Chemical Reactions. J. Comput. Phys. 1976, 22 (4), 403-434. 
(70) Gillespie, D. T. Exact Stochastic Simulation of Coupled Chemical Reactions. J. Phys. Chem. 1977, 81 (25), 2340-2361.

(71) Luo, Z.-H.; Shi, D.-P.; Zhu, Y. Multiple Active Site Monte Carlo Model for Heterogeneous Ziegler-Natta Propylene Polymerization. J. Appl. Polym. Sci. 2010, 115 (5), 2962-2970.

(72) Van Steenberge, P. H. M.; D’hooge, D. R.; Reyniers, M.-F.; Marin, G. B. Improved Kinetic Monte Carlo Simulation of Chemical Composition-Chain Length Distributions in Polymerization Processes. Chem. Eng. Sci. 2014, 110, 185-199.

(73) Mastan, E.; Li, X.; Zhu, S. Modeling and Theoretical Development in Controlled Radical Polymerization. Prog. Polym. Sci. 2015, 45, 71-101.

(74) Hagberg, A. A.; Schult, D. A.; Swart, P. J. Exploring Network Structure, Dynamics, and Function Using NetworkX. 2008, 5.

(75) Ferrini, P.; Rinaldi, R. Catalytic Biorefining of Plant Biomass to Non-Pyrolytic Lignin Bio-Oil and Carbohydrates through Hydrogen Transfer Reactions. Angew. Chem. 2014, 126 (33), 8778-8783.

(76) Phongpreecha, T.; Hool, N. C.; Stoklosa, R. J.; Klett, A. S.; Foster, C. E.; Bhalla, A.; Holmes, D.; Thies, M. C.; Hodge, D. B. Predicting Lignin Depolymerization Yields from Quantifiable Properties Using Fractionated Biorefinery Lignins. Green Chem. 2017, 19 (21), 5131-5143.

(77) Shuai, L.; Amiri, M. T.; Questell-Santiago, Y. M.; Héroguel, F.; Li, Y.; Kim, H.; Meilan, R.; Chapple, C.; Ralph, J.; Luterbacher, J. S. Formaldehyde Stabilization Facilitates Lignin Monomer Production during Biomass Depolymerization. Science 2016, 354 (6310), 329-333.

(78) Parsell, T.; Yohe, S.; Degenstein, J.; Jarrell, T.; Klein, I.; Gencer, E.; Hewetson, B.; Hurt, M.; Kim, J. I.; Choudhari, H.; et al. A Synergistic Biorefinery Based on Catalytic Conversion of Lignin Prior to Cellulose Starting from Lignocellulosic Biomass. Green Chem. 2015, 17 (3), 1492-1499.

(79) Luo, H.; M. Abu-Omar, M. Lignin Extraction and Catalytic Upgrading from Genetically Modified Poplar. Green Chem. 2018, 20 (3), 745-753.

(80) Anderson, E. M.; Stone, M. L.; Katahira, R.; Reed, M.; Muchero, W.; Ramirez, K. J.; Beckham, G. T.; RománLeshkov, Y. Differences in S/G Ratio in Natural Poplar Variants Do Not Predict Catalytic Depolymerization Monomer Yields. Nat. Commun. 2019, 10 (1), 2033.

(81) Vermaas, J. V.; Dixon, R. A.; Chen, F.; Mansfield, S. D.; Boerjan, W.; Ralph, J.; Crowley, M. F.; Beckham, G. T. Passive Membrane Transport of Lignin-Related Compounds. Submitted.

(82) Dima, O.; Morreel, K.; Vanholme, B.; Kim, H.; Ralph, J.; Boerjan, W. Small Glycosylated Lignin Oligomers Are Stored in Arabidopsis Leaf Vacuoles. Plant Cell 2015, 27 (3), 695-710.

(83) Anahashi, M. T.; Higuchi, T. Dehydrogenative Polymerization of Monolignols by Peroxidase and H202 in a Dialysis Tube. I. Preparation of Highly Polymerized DHPs. 1981, No. 67, 15.

(84) Terashima, N.; Atalla, R. H.; Ralph, S. A.; Landucci, L. L.; Lapierre, C.; Monties, B. New Preparations of Lignin Polymer Models under Conditions That Approximate Cell Wall Lignification. I. Synthesis of Novel Lignin Polymer Models and Their Structural Characterization by ${ }^{13}$ C NMR. Holzforschung 1995, 49 (6), 521527.

(85) Galkin, M. V.; Samec, J. S. M. Lignin Valorization through Catalytic Lignocellulose Fractionation: A Fundamental Platform for the Future Biorefinery. ChemSusChem 2016, 9 (13), 1544-1558.

(86) Sake, B.; Argyropoulos, D. S.; Beinhoff, O.; Faix, O. A Comparison of Lignin Polymer Models (DHPs) and Lignins by 31P NMR Spectroscopy. Phytochemistry 1996, 43 (2), 499-507.

(87) Nimz, H. H.; Lüdemann, H.-D. Kohlenstoff-13-NMR-Spektren von Ligninen, 6. Lignin- Und DHP-Acetate. Holzforsch. - Int. J. Biol. Chem. Phys. Technol. Wood 1976, 30 (2), 33-40.

(88) Nimz, H.; Mogharab, I.; Lüdemann, H.-D. 13C-kernresonanzspektren von ligninen, 3. Vergleich von fichtenlignin mit künstlichem lignin nach freudenberg. Makromol. Chem. 1974, 175 (9), 2563-2575.

(89) Chen, F.; Tobimatsu, Y.; Havkin-Frenkel, D.; Dixon, R. A.; Ralph, J. A Polymer of Caffeyl Alcohol in Plant Seeds. Proc. Natl. Acad. Sci. 2012, 109 (5), 1772-1777.

(90) Berstis, L.; Elder, T.; Crowley, M.; Beckham, G. T. Radical Nature of C-Lignin. ACS Sustain. Chem. Eng. 2016, 4 (10), 5327-5335.

(91) Carlos del Río, J.; Rencoret, J.; Gutiérrez, A.; Kim, H.; Ralph, J. Hydroxystilbenes Are Monomers in Palm Fruit Endocarp Lignins. Plant Physiol. 2017, 174 (4), 2072-2082. 
(92) Stone, M. L.; Anderson, E. M.; Meek, K. M.; Reed, M.; Katahira, R.; Chen, F.; Dixon, R. A.; Beckham, G. T.; Román-Leshkov, Y. Reductive Catalytic Fractionation of C-Lignin. ACS Sustain. Chem. Eng. 2018, 6 (9),

(93) Li, Y.; Shuai, L.; Kim, H.; Motagamwala, A. H.; Mobley, J. K.; Yue, F.; Tobimatsu, Y.; Havkin-Frenkel, D.; Chen, F.; Dixon, R. A.; et al. An "Ideal Lignin" Facilitates Full Biomass Utilization. Sci. Adv. 2018, 4 (9), eaau2968. 


\section{Supporting Information:}

\section{Methods}

\section{Implementation Details}

4 Our version of the Gillespie algorithm for studying lignification was implemented in Python 3.6.6, ${ }^{1}$ using NumPy $5 \quad$ 1.15.1, ${ }^{2}$ SciPy 1.1.0, ${ }^{3}$ MatPlotLib 2.2.2, ${ }^{4}$ and RDKit 2018.03.4.0. ${ }^{5}$ Most of the developed code was then used in 6 Jupyter notebooks running on the IPython 6.5.0 kernel. ${ }^{6}$ The installation and use of the source code can be found 7 from the GitHub repository (https://github.com/michaelorella/lignin-kmc). ${ }^{7}$

\section{Computational performance}

9 An analysis of the algorithm performance using the built-in time package in Python demonstrated $O\left(N^{2.54}\right)$ scaling. The 10 scaling analysis ran simulations under a set of constant conditions (e.g., S to G ratio of 1 and total simulation time) but 11 varied the number of monomers between 50 and 1000 with constant spacing. Due to the stochastic nature of the 12 simulation, the execution time is expected to vary slightly from run to run, so each simulation was repeated 5 times 13 while recording the average clock time with error bars corresponding to one standard deviation. The resulting times 14 were plotted as a function of the number of monomers included in the simulation, and the resulting curve was fit to a 15 generic power law model of the form $t=a N^{b}$, where $a$ and $b$ are the fitting parameters, and $b$ represents the scaling of the algorithm. The fitted curve together with the measured data are shown in Figure S1.

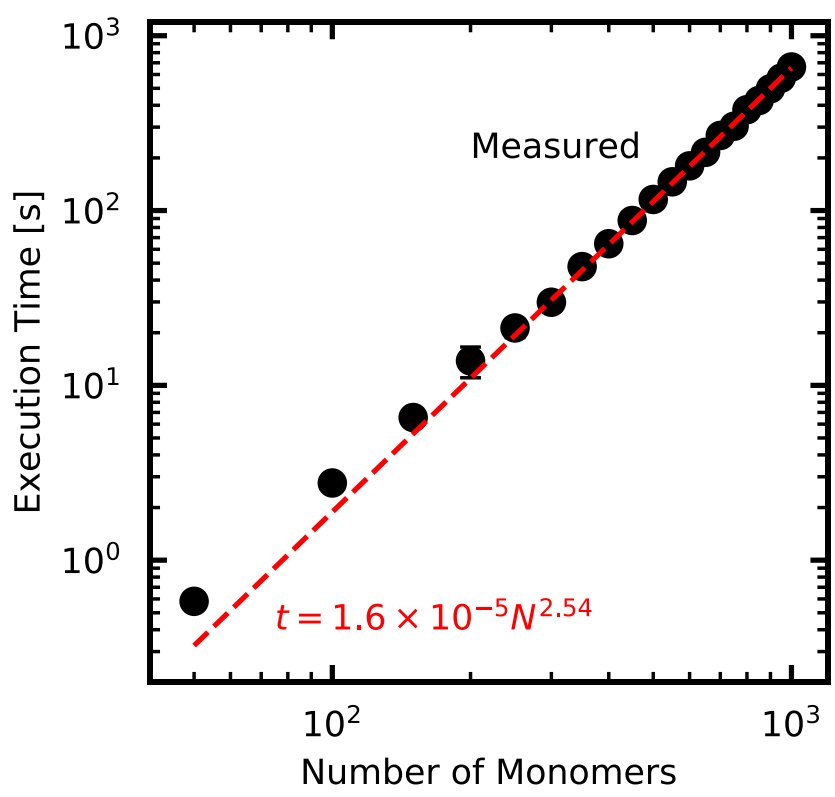

Figure S1: Computational time complexity of Gillespie algorithm implemented through the described methodology. Black dots indicate the measured wall time to execute a simulation with the specified number of monomers, averaged over 5 runs, while the red dashed line is the power law fit to the measurements.

21 This performance can be rationalized by examining the specific implementation of the algorithm. In this case, we 
the number of monomers. However, the strong dependence on number of events combined with the understanding that the number of events recorded in the simulation should increase in a combinatorial manner, thus the superlinear behavior of our algorithm appears reasonable. It should be noted that while the maximum number of events scales in a combinatorial manner, we do not observe combinatorial scaling because only a small subset of events from the previous iteration are repeated as opposed to a reconstruction of the entire set of events at every iteration.

While the computational cost will be minimized by including fewer monomers in the simulation, this will also compromise the integrity of the simulation. To evaluate the tradeoff between the computational cost and simulation accuracy, we attempt to observe the convergence of the measured property means and variance of the calculated distributions, as shown in Figure S2. These data clearly show that as the number of monomers included in the simulation increase, there is not a significant effect on the bond content, but there is a slight decrease in the mean monomer yield that is observed. Meanwhile, there is a significant decrease in the variance within the observed bond distributions that approaches 0 , while a similar decrease in the monomer yield variance that approaches a finite value. Based on these observations, for all simulations shown in the main text, 500 monomers are used to minimize the expected variance while simultaneously bounding the computational cost.
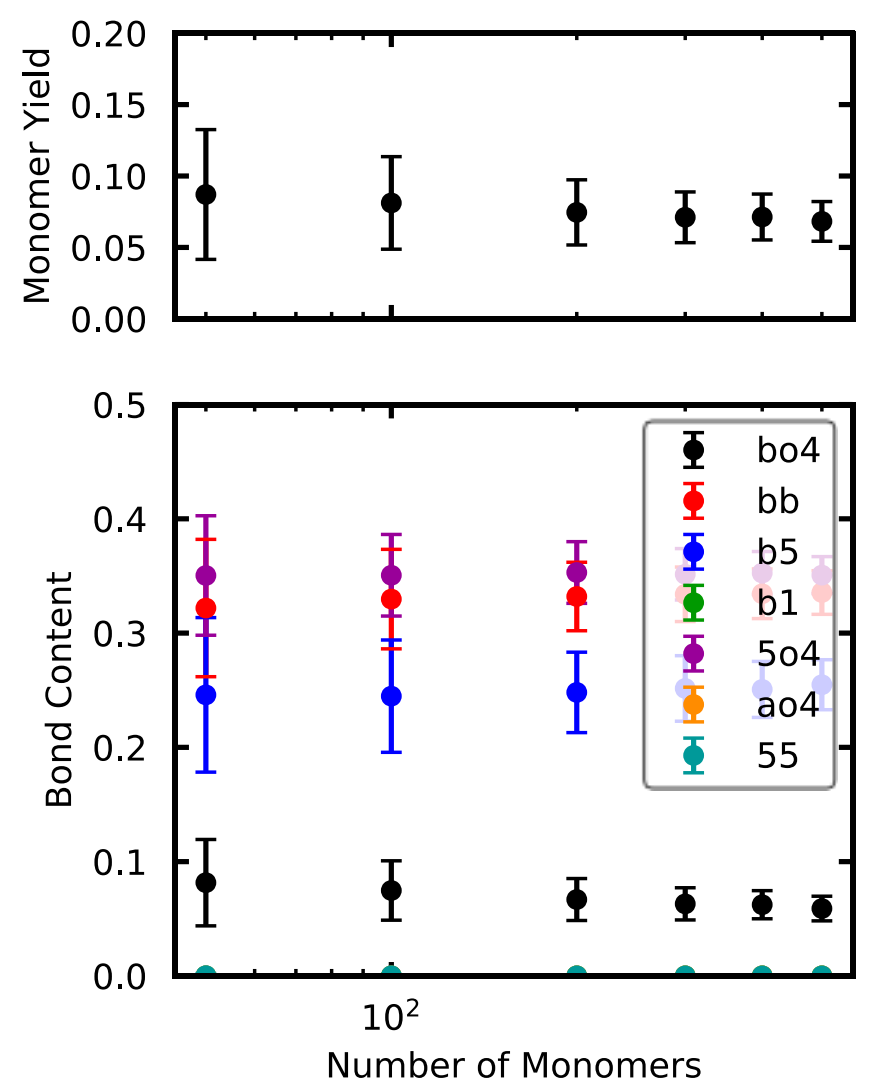

Figure S2: Convergence of algorithm as a function of number of monomers included in the simulation. The effect of changing the number of monomers in the simulation on the depolymerization monomer yields (Top) and bond contents (Bottom). As the number 
of monomers increases, the variance decreases, but the means don't appear to change significantly. Error bars are one standard deviation.

\section{Conversion to MolFile format}

To better understand the simulation output, we developed a method to output 2D representations of the generated lignin using the RDKit package in Python. ${ }^{5}$ Before this package could be used to obtain $2 \mathrm{D}$ chemical structures, we first needed to convert the lignin data structure into the traditional MolFile V3000 format. ${ }^{8}$ To perform this conversion, we must convert the graph of monomers to a graph containing all atoms. This conversion occurs in the following steps: (1) encode MolFiles for the individual monomer types, (2) iterate through monomers stitching the individual files together and updating indices in the aggregate MolFile, (3) iterate through the adjacency matrix to add connections between the monomers and modifying any other parts of the monomer that might be changed by bonding. Once the final MolFile has been created, RDKit or other chemical drawing tools can be used directly to compute the 2D coordinates for each of the atoms (attempting to reduce overlap of the drawn structure), and then finally the structure can be drawn. The strings used in step (1) and details of the stitching and necessary modifications can be found in the file "visualization.py" of the source code.

\section{Conversion to other formats}

To facilitate molecular visualization and simulation, other output formats have also been implemented. A direct export pipeline to LigninBuilder converts the internal graph representation from LIGNIN-KMC into input for the molecular modeling tool psfgen by directly reading the non-zero entries of the adjacency matrix and translating this into specific lignin linkages. From that point, 3D coordinates can be generated via LigninBuilder, resulting in a structure read for atomic simulation. To visualize large structures, we have also translated native LIGNIN-KMC output into a simplified graph representation of lignin leveraging the NetworkX python library, ${ }^{9}$ with individual monomers represented as nodes and linkages represented as edges within the graph.

\section{Modified Depth First Search for Connected Subgraph Identification}

The problem of identifying connected subgraphs in a graph is well-studied in graph theory. Hopcroft and Tarjan report a method of using a depth first search (DFS) algorithm to identify these connected components. ${ }^{10}$ Based on their algorithm, the following steps are performed:

1) The remaining nodes in the graph are set to all of the nodes on the graph and the current node is set to 0

2) An empty stack is instantiated to keep track of the nodes that could be visited on this connected component

3) An empty set is instantiated that will contain the nodes in this component

4) The current node is removed from the list of remaining nodes and added to the current component

5) The current node is expanded and all connections that have yet to be visited are added to the stack so long as they are not already on the stack 
6) The current node is updated to be the top of the stack if the stack has remaining elements or to be the next remaining node if there are no more elements on the stack. In the latter case, a new connected component is begun.

75 7) Repeat from (4) until there are no remaining nodes

76 When all of the independent connected components have been identified, it is trivial to measure the length of set, which 77 determines the degree $N$-mer that each connected fragment exists as.

\section{$78 \quad$ Rate Constants for S/G Lignin}

79 Activation energies reported by Gani et al. ${ }^{11}$

80 Table 1: Energy parameters used for the kinetic modeling of lignification that were obtained from DFT calculations. The linked 81 species refers to the type of monomer being bound, while the chain size refers to whether a bond is occurring between a monomer 82 (1) or a growing chain (2+).

\begin{tabular}{|c|c|c|c|}
\hline $\begin{array}{l}\text { Bond } \\
\text { Type }\end{array}$ & $\begin{array}{l}\text { Linked } \\
\text { Species }\end{array}$ & Chain Sizes & $\begin{array}{c}\Delta G_{j}^{\dagger} \\
\text { (kcal/mol) }\end{array}$ \\
\hline \multirow{2}{*}{504} & $(\mathrm{G}, \mathrm{G})$ & $\begin{array}{c}(1,1) \\
(1 / 2+, 2+/ 1) \\
(2+, 2+)\end{array}$ & $\begin{array}{c}11.2 \\
14.6 \\
4.4\end{array}$ \\
\hline & $(\mathrm{S}, \mathrm{G})$ & $\begin{array}{c}(1,1) \\
(1 / 2+, 2+/ 1) \\
(2+, 2+)\end{array}$ & $\begin{array}{c}10.9 \\
14.6 \\
4.4\end{array}$ \\
\hline 55 & $(\mathrm{G}, \mathrm{G})$ & $\begin{array}{c}(1,1) \\
(1 / 2+, 2+/ 1) \\
(2+, 2+)\end{array}$ & $\begin{array}{c}12.5 \\
15.6 \\
3.8\end{array}$ \\
\hline$\beta 5$ & $(\mathrm{G} / \mathrm{G}, \mathrm{G} / \mathrm{S})$ & $\begin{array}{c}(1,1) \\
(1 / 2+/ 2+, \\
2+/ 1 / 1)\end{array}$ & $\begin{array}{l}5.5 \\
5.8\end{array}$ \\
\hline \multirow{2}{*}{$\beta \beta$} & $(\mathrm{G} / \mathrm{S}, \mathrm{G} / \mathrm{S})$ & $\begin{array}{l}(1 / 2+/ 1 / 2+ \\
1 / 1 / 2+/ 2+)\end{array}$ & 5.2 \\
\hline & $(\mathrm{G} / \mathrm{S}, \mathrm{S} / \mathrm{G})$ & $\begin{array}{l}(1 / 2+/ 1 / 2+ \\
1 / 1 / 2+/ 2+)\end{array}$ & 6.5 \\
\hline \multirow[t]{2}{*}{$\beta 04$} & $(\mathrm{G}, \mathrm{G})$ & $\begin{array}{c}(1,1) \\
(2+/ 1 / 2+, \\
1 / 2+/ 2+)\end{array}$ & $\begin{array}{l}6.3 \\
6.2\end{array}$ \\
\hline & $(\mathrm{S}, \mathrm{G})$ & $(1,1)$ & 9.1 \\
\hline
\end{tabular}




\begin{tabular}{|c|c|c|c|}
\hline & & $\begin{array}{l}(2+/ 1 / 2+ \\
1 / 2+/ 2+)\end{array}$ & 6.2 \\
\hline & $(\mathrm{G}, \mathrm{S})$ & $\begin{array}{c}(1,1) \\
(2+/ 1 / 2+ \\
1 / 2+/ 2+)\end{array}$ & $\begin{array}{l}8.9 \\
6.2\end{array}$ \\
\hline & $(\mathrm{S}, \mathrm{S})$ & $\begin{array}{c}(1,1) \\
(2+/ 1 / 2+ \\
1 / 2+/ 2+)\end{array}$ & $\begin{array}{r}9.8 \\
10.4\end{array}$ \\
\hline$\alpha O 4$ & $\begin{array}{l}\text { (G/S/G/S, } \\
\text { G/G/S/S })\end{array}$ & $\begin{array}{l}(1 / 2+/ 1 / 2+ \\
1 / 1 / 2+/ 2+)\end{array}$ & 20.7 \\
\hline \multirow{4}{*}{$\beta 1$} & $(\mathrm{G}, \mathrm{G})$ & $\begin{array}{l}(1 / 2+/ 2+ \\
2+/ 1 / 2+)\end{array}$ & 9.6 \\
\hline & $(\mathrm{S}, \mathrm{G})$ & $\begin{array}{l}(1 / 2+/ 2+ \\
2+/ 1 / 2+)\end{array}$ & 11.7 \\
\hline & $(\mathrm{G}, \mathrm{S})$ & $\begin{array}{l}(1 / 2+/ 2+ \\
2+/ 1 / 2+)\end{array}$ & 10.7 \\
\hline & $(\mathrm{S}, \mathrm{S})$ & $\begin{array}{l}(1 / 2+/ 2+ \\
2+/ 1 / 2+)\end{array}$ & 11.9 \\
\hline Hydration & $\begin{array}{l}\mathrm{G} \\
\mathrm{S}\end{array}$ & $\begin{array}{l}1 / 2+ \\
1 / 2+\end{array}$ & $\begin{array}{l}11.1 \\
11.7\end{array}$ \\
\hline \multirow{2}{*}{ Oxidation } & $\mathrm{G}$ & $\begin{array}{c}1 \\
2+\end{array}$ & $\begin{array}{l}0.9 \\
6.3\end{array}$ \\
\hline & $S$ & $\begin{array}{c}1 \\
2+\end{array}$ & $\begin{array}{l}0.6 \\
2.2\end{array}$ \\
\hline
\end{tabular}

\section{Rate Constants for C Lignin}

84 Activation energies were computed for coupling of caffeoyl alcohol monomers according to the protocol in Gani et 85 al. $^{11}$

\begin{tabular}{c|c|c} 
Bond Type & Chain Sizes & $\begin{array}{c}\Delta G_{j}^{\ddagger} \\
(\mathbf{k c a l} / \mathbf{m o l})\end{array}$ \\
\hline \hline 504 & $\begin{array}{c}(1 / 1 / 2+/ 2+, \\
1 / 2+/ 1 / 2+)\end{array}$ & 11.9
\end{tabular}




\begin{tabular}{c|c|c}
\hline 55 & $\begin{array}{c}(1 / 1 / 2+/ 2+, \\
1 / 2+/ 1 / 2+)\end{array}$ & 10.6 \\
\hline \multirow{2}{*}{$\beta 5$} & $\begin{array}{c}(1,1) \\
(1 / 2+/ 2+, \\
2+/ 1 / 1)\end{array}$ & 5.8 \\
\hline$\beta \beta$ & $(1 / 2+/ 1 / 2+$, & 7.2 \\
\hline$\beta 04$ & $1 / 1 / 2+/ 2+)$ & 4.9 \\
\hline$\beta 1$ & $(1,1)$ & 1.3 \\
\hline$\alpha 04$ & $1 / 2+/ 2+)$ & 20.7 \\
\hline Hydration & $(1 / 2+/ 1 / 2+$, & \\
\hline Oxidation & $1 / 1 / 2+/ 2+)$ & 9.6 \\
\hline
\end{tabular}

86

$87 \quad$ Results 

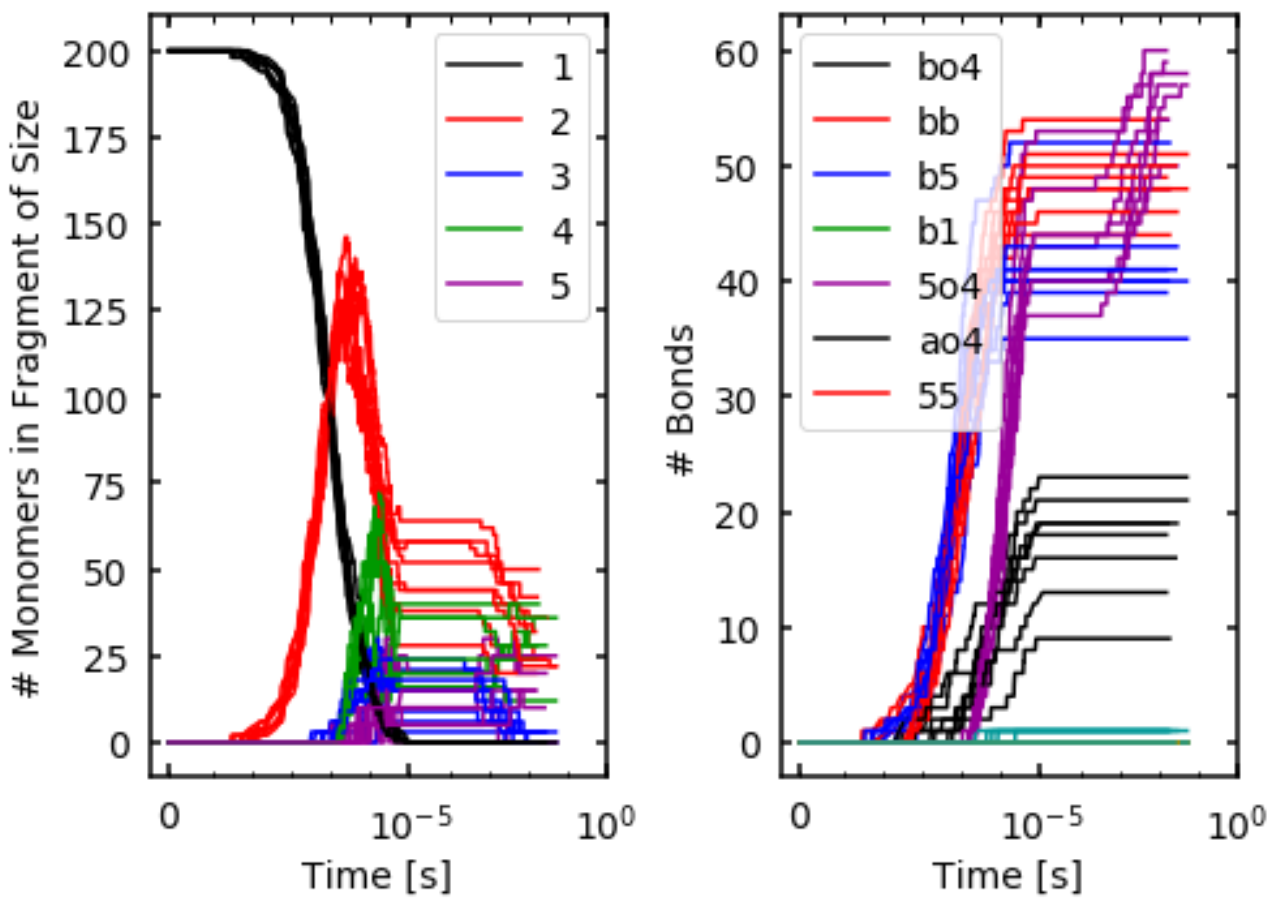

90 Figure S3: Dynamics of a typical LIGNIN-KMC simulation beginning with 200 monomers initially, no monomers added to the simulation, with an $S / G$ ratio of 1 , for a total time of 10 kiloseconds. These traces show 8 repeated simulations where the same initial conditions are used, but the random number selection results in a different evolution of the simulation state.
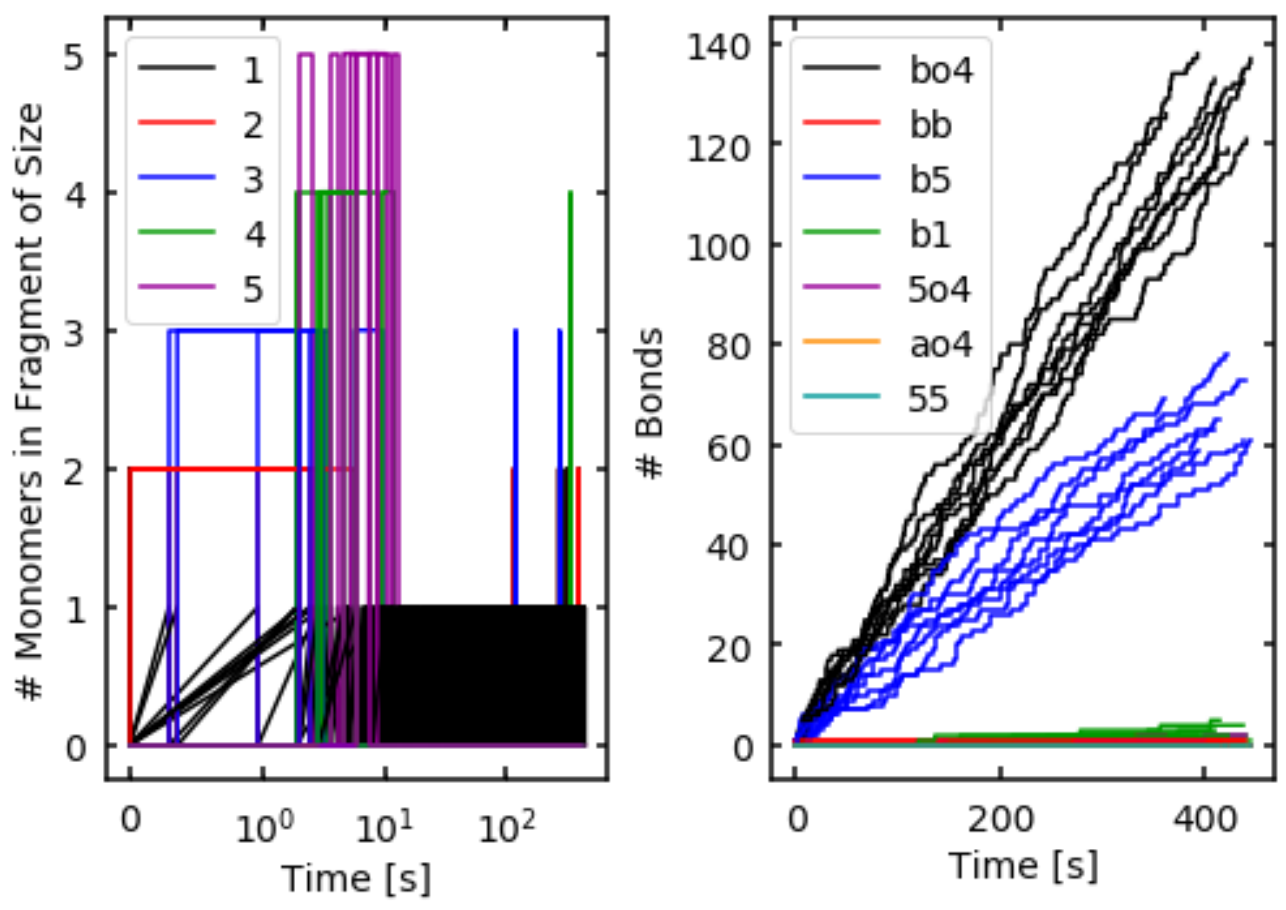
Figure S4: Dynamics of a typical LIGNIN-KMC simulation beginning with 2 monomers initially, monomers added to the simulation at a rate of 1 monomers per second capped at a maximum of 200 in the simulation at a time, with an $S / G$ ratio of 1 , for a total time of 10 kiloseconds. These traces show 8 repeated simulations where the same initial conditions are used, but the random number 98 selection results in a different evolution of the simulation state.

99
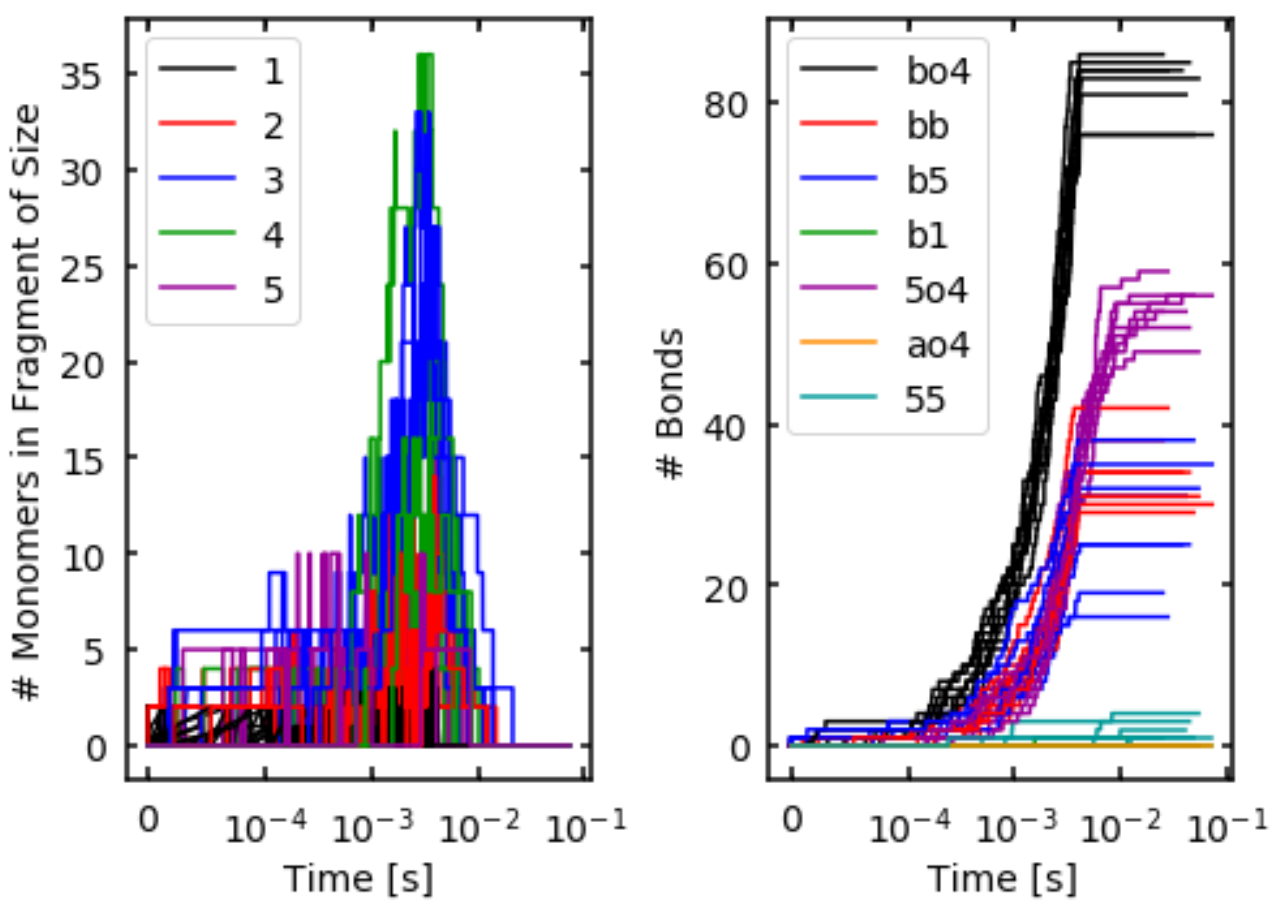

Figure S5: Dynamics of a typical LIGNIN-KMC simulation beginning with 2 monomers initially, monomers added to the simulation at a rate of $10^{5}$ monomers per second capped at a maximum of 200 in the simulation at a time, with an $S / G$ ratio of 1 , for a total time of 10 kiloseconds. These traces show 8 repeated simulations where the same initial conditions are used, but the random number selection results in a different evolution of the simulation state. 


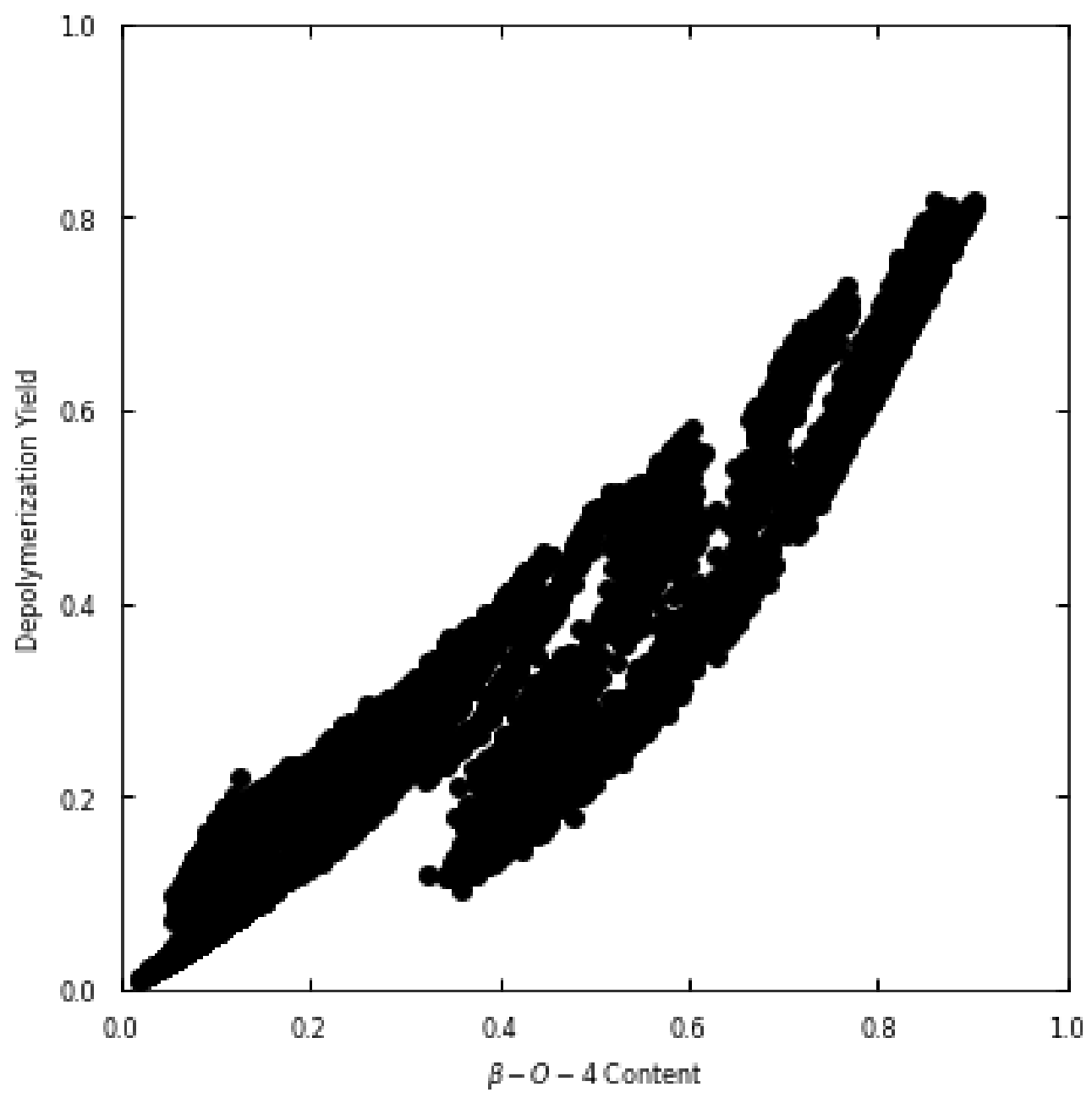

Figure S6: An alternative visualization of the data presented in Figure 5, where all of the simulations are represented as points on this plot. The $\beta O 4$ content and monomer yield are extracted from each simulation and then plotted against each other to observe the existence of correlation.

\section{References}

111 (1) Python 3.6.8 https://docs.python.org/3.6/ (accessed Jan 14, 2019).

112 (2) Oliphant, T. A Guide to NumPy; Trelgol Publishing: USA, 2006.

113 (3) Jones, E.; Oliphant, T.; Peterson, P.; others. SciPy: Open Source Scientific Tools for Python. 2001.

114 (4) Hunter, J. D. Matplotlib: A 2D Graphics Environment. Comput. Sci. Eng. 2007, 9 (3), 90-95.

115 (5) RDKit: Open-source cheminformatics http://www.rdkit.org.

116 (6) Perez, F.; Granger, B. E. IPython: A System for Interactive Scientific Computing. Comput. Sci. Eng. 2007, 9 (3), $117 \quad 21-29$.

118 (7) Orella, M. Michaelorella/Lignin-Kmc.

119 (8) CTfile Formats; Symyx Solutions, Inc., 2010.

120 (9) Hagberg, A. A.; Schult, D. A.; Swart, P. J. Exploring Network Structure, Dynamics, and Function Using NetworkX. 2008, 5.

(10) Hopcroft, J.; Tarjan, R. Algorithm 447: Efficient Algorithms for Graph Manipulation. Commun. ACM 1973, 16 (6), 372-378. 
(11) Gani, T.; Orella, M.; Anderson, E.; Stone, M.; Brushett, F.; Beckham, G.; Román-Leshkov, Y. Quantum Mechanical Calculations Suggest That Lignin Polymerization Is Kinetically Controlled. 2018. 
